\title{
Weakly nonlinear oscillations of nearly inviscid axisymmetric liquid bridges
}

\author{
By JOSÉ A. NICOLÁS AND JOSÉ M. VEGA \\ ETSI Aeronáuticos, Universidad Politécnica de Madrid, 28040-Madrid, Spain
}

(Received 9 August 1995 and in revised form 22 May 1996)

A weakly nonlinear analysis is presented of the small oscillations of nearly inviscid liquid bridges subjected to almost resonant axial vibrations of the disks. An amplitude equation is derived for the evolution of the complex amplitude of the oscillations that exhibits hysteresis and period doublings. We also analyse the steady streaming in the bulk forced by the oscillatory boundary layers near the disks; the boundary layer near the free surface produces no forcing terms. In particular some experimentally observed patterns are explained, and some new, non-observed ones are predicted. We conclude that the structure of this steady flow is not the appropriate one to counterbalance steady thermocapillary convection, but our results indicate how to get the desired counterbalancing effect.

\section{Introduction}

The stability and dynamics of liquid columns and jets were considered in the pioneering work by Plateau $(1849,1863)$ and Rayleigh $(1879,1892)$, after the earlier basic analyses on capillary interfaces by Young (1805) and Laplace (1805). In the last twenty years the interest in these configurations has increased due to their applications in some industrial processes and natural phenomena. In particular, liquid bridges are of interest in materials processing (Preiser, Schwabe \& Sharman 1983; Kamotani, Ostrach \& Vargas 1984; Brown 1988) and have been observed in flow through porous media (Melrose 1966; Zasadzinski et al. 1987) and particulates agglomeration (Chen, Tsamopoulos \& Good 1992); other applications include the experimental measurement of viscosity and surface tension (Tsamopoulos, Chen \& Borkar 1992), and their use as accelerometers.

Resonance frequencies were determined experimentally by Fowle, Wang \& Strong (1979) and Mollot et al. (1993) for isolated liquid bridges and by Meseguer (1983) and Sanz (1985) for a liquid bridge immersed in a bath of another liquid of nearly equal density. On the theoretical side, most results in the literature were concerned with one-dimensional approximations (Meseguer 1983; Rivas \& Meseguer 1984; Zhang \& Alexander 1990; Meseguer \& Perales 1991; Perales \& Meseguer 1992) that became very popular for liquid bridges and jets ten years ago; in addition to other limitations (Schulkes $1993 a, b$ ), the validity of one-dimensional models is restricted to slender bridges. Among the exceptions to this rule concerning linear approximations, let us mention some analyses of the inviscid case (Sanz 1985; Sanz \& Díez 1989), of the limits of large and small capillary Reynolds number (Borkar \& Tsamopoulos 1991; Nicolás 1992), and the (fairly expensive) numerical calculations for finite Reynolds numbers by Tsamopoulos et al. (1992). In particular, a boundary layer approximation was 
used by Borkar \& Tsamopoulos (1991) to calculate a first correction to the natural frequencies and a first approximation of the damping rates, that only accounted for viscous dissipation in the Stokes boundary layers near the disks supporting the bridge. Unfortunately, although these results are asymptotically correct, they provide a poor approximation to the damping rate, except for unrealistically large values of the capillary Reynolds number, as shown by Higuera, Nicolás \& Vega (1994), who calculated a second approximation (accounting for viscous dissipation both in the Stokes boundary layers and in the bulk) to obtain quite good results for moderately large Reynolds numbers; see also Nicolás \& Vega (1996) for a comparison with almost exact results obtained by a semi-analytical method that allows cheap computations for arbitrary values of the capillary Reynolds number.

The only theoretical work in the literature concerning nonlinear mechanical oscillations of non-inviscid liquid bridges, not using one-dimensional approximations, is a recent paper by Chen \& Tsamopoulos (1993), who analysed fully nonlinear oscillations by direct numerical simulation. They considered only moderately large values of the capillary Reynolds number, $C^{-1} \leqslant 50$; their computations are seemingly too expensive for larger values of $C^{-1}$. They showed in particular how nonlinear terms affect quantitatively the natural frequencies and damping rates.

In this paper we consider the weakly nonlinear response of an axisymmetric, nearly inviscid liquid bridge to forced vibrations of the disks, of appropriately small amplitude and nearly resonant frequency (i.e. close to a natural frequency of the bridge). We shall obtain the coefficients of a well-known cubic canonical amplitude equation (that applies here and in related almost-conservative problems) in terms of the slenderness of the bridge and the inviscid mode being excited. This will be done in $\$ 3$ where, according to the results by Higuera et al. (1994), we shall use a two-terms approximation of the damping rate; this will make our perturbation scheme somewhat non-standard. The amplitude equation will be analysed in $\$ 4$ to show that it exhibits hysteresis (as is well-known) and also relaxation oscillations and period doublings when both disks are vibrated with slightly different frequencies. Section 5 will be devoted to the analysis of the streaming flow in the bulk induced by the oscillatory viscous boundary layers, intending to see whether this flow could annihilate stationary thermocapillary flows, as in the recent experiment by Anilkumar et al. (1993). The result could be of great interest in, e.g., materials processing in microgravity, for thermocapillary flows are always present in the melt when applying the float-zone technique for unidirectional semiconductor crystal growth, and are currently assumed to be responsible for the formation of undesirable non-uniformities in dopant distribution and crystal striations (see, e.g., Jurish \& Löser 1990). We shall not get a completely satisfactory answer to that question, but our analysis will show how to get the appropriate counterbalancing effect, namely the disks should be vibrated with either higher frequencies (as in the experiment by Anilkumar et al. 1993, and in Nicolás, Rivas \& Vega 1996b) or non-resonant frequencies (as in Nicolás, Rivas \& Vega 1996a). As a by-product we shall explain in $\$ 5$ some steady streaming flow patterns observed by Mollot et al. (1993), and predict some new ones. In order to illustrate our results, two examples will be briefly considered in $\S 6$, in the space of laboratory dimensional parameters. Finally, some concluding remarks will be drawn in $\S 7$.

\section{Formulation}

In order to analyse mechanical vibrations and the resulting streaming flow we consider a liquid bridge of length $L$, held by surface tension forces between two 
parallel, circular, coaxial disks of equal radii $R$. The volume of the liquid equals that of the space in the cylinder bounded by the disks. In addition, we neglect gravity and assurne that the density $\rho$ and kinematic viscosity $v$ of the liquid and the surface tension $\sigma$ are uniform and constant, and such that the capillary Reynolds number, $C^{-1}=\left(\sigma R / \rho v^{2}\right)^{1 / 2}$, is large, and that $\rho$ and $\rho v$ are large compared to the density and viscosity of the surrounding gas (then the gas does not affect the dynamics of the liquid bridge). Finally, the free surface of the liquid is anchored at the borders of the disks, and the disks are vibrating independently in the axial direction.

Under the assumptions above we use $R$ and the capillary time $\left(\rho R^{3} / \sigma\right)^{1 / 2}$ as characteristic length and time for non-dimensionalization to write the governing equations (continuity and momentum conservation) and boundary conditions (nonslipping at the disks, smoothness of the pressure and velocity fields at the axis of symmetry, kinematic compatibility and tangential and normal stress balances at the free surface) as

$$
\begin{aligned}
& u_{\mathrm{r}}+r^{-1} u+w_{z}=0 \\
& u_{t}+w\left(u_{z}-w_{r}\right)=-q_{r}+C\left(u_{r r}+r^{-1} u_{r}-r^{-2} u+u_{z z}\right) \text {, } \\
& w_{t}+u\left(w_{r}-u_{z}\right)=-q_{z}+C\left(w_{r r}+r^{-1} w_{r}+w_{z z}\right) \text {, } \\
& u=0, \quad w=h_{ \pm}^{\prime}(t) \quad \text { at } \quad z= \pm A+h_{ \pm}(t), \\
& u=w_{r}=q_{r}=0 \quad \text { at } r=0 \text {, } \\
& u=f_{t}+f_{z} w \quad \text { at } r=f \text {, } \\
& \left(w_{r}+u_{z}\right)\left(1-f_{z}^{2}\right)+2\left(u_{r}-w_{z}\right) f_{z}=0 \quad \text { at } r=f, \\
& q-\frac{u^{2}+w^{2}}{2}+\frac{f f_{z z}-1-f_{z}^{2}}{f\left(1+f_{z}^{2}\right)^{3 / 2}}=2 C \frac{u_{r}-\left(w_{r}+u_{z}\right) f_{z}+w_{z} f_{z}^{2}}{1+f_{z}^{2}} \\
& f=1 \quad \text { at } \quad z= \pm A+h_{ \pm}(t),
\end{aligned}
$$$$
\text { at } r=f \text {, }
$$

with appropriate initial conditions. Here a cylindrical coordinate system $(r, \varphi, z)$ is used with the origin midway between the disks, the axis of symmetry as the $z$-axis and associated unit vectors $e_{r}, e_{\varphi}$ and $e_{z}$. The velocity field is $u e_{r}+w e_{z}$ and for convenience the problem is written in terms of the stagnation pressure, $q=$ pressure $+\left(u^{2}+w^{2}\right) / 2$; the shape of the interface is given by $r=f(z, t)$ and $A=L / 2 R$ is the slenderness of the bridge. Notice that the total volume of the liquid is conserved (as a consequence of (2.1), (2.4)-(2.6)) and, according to the assumption above, equals $2 \pi A$, i.e.

$$
\int_{-\Lambda+h_{-}}^{\Lambda+h_{+}} f(z, t)^{2} \mathrm{~d} z=2 \Lambda .
$$

Finally the functions $h_{ \pm}$are assumed to be given by

$$
h_{ \pm}(t)=\mu \beta_{ \pm} \exp \left[\mathrm{i}\left(\Omega+\omega_{ \pm} \delta\right) t\right]+\text { c.c. }
$$

where c.c. stands for the complex conjugate, $\Omega>0$ is a given natural frequency of the bridge in the inviscid limit, $\omega_{-}$and $\omega_{+}$are real constants, $\beta_{-}$and $\beta_{+}$are complex constants and $\mu$ and $\delta$ are real, positive parameters. We shall consider (2.1)-(2.9) in the limit

$$
C \ll 1, \quad \mu \ll 1 \quad \text { and } \delta \ll 1,
$$

without making at this stage further assumptions concerning the relative orders of magnitude of $C, \mu$ and $\delta$.

The formulation above deals with the purely mechanical problem, which is the main object of the paper. But in order to analyse the combined effect of vibrations 


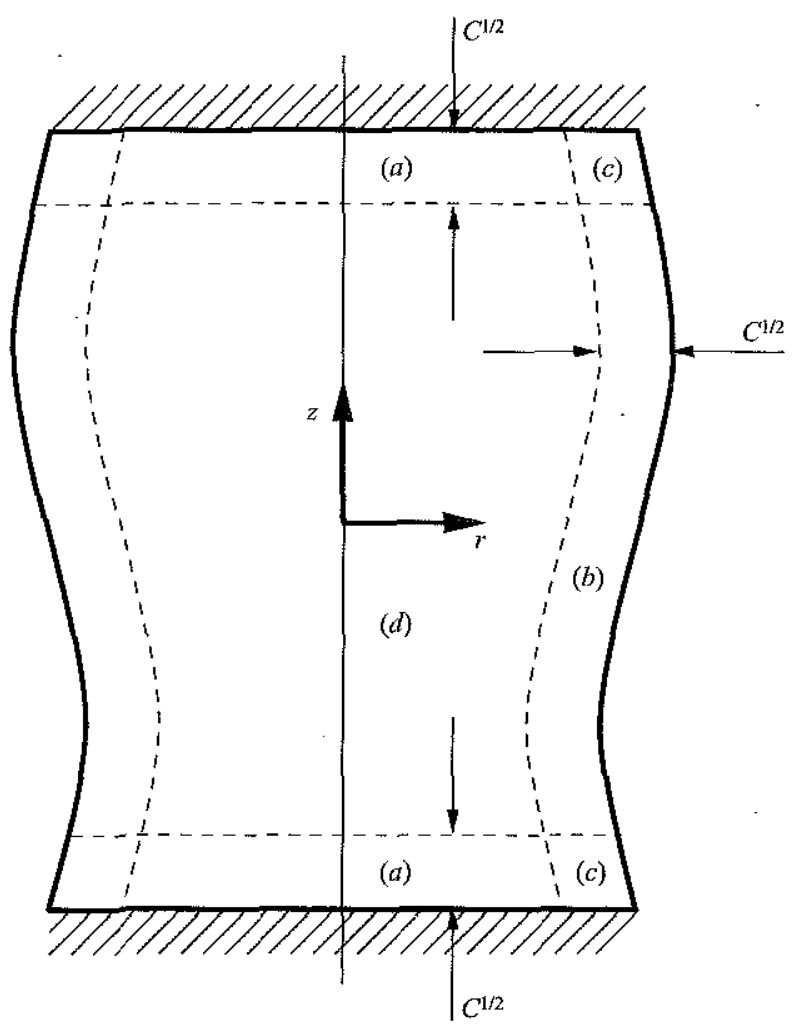

Figure 1. Sketch of the four regions in the liquid bridge.

and thermocapillary stress in non-isothermal conditions, in $\$ 5.1$ we shall add (i) a thermocapillary stress to the tangential stress balance (2.7), and (ii) the energy equation with appropriate boundary conditions.

\section{Derivation of the amplitude equation $\dagger$}

The weakly nonlinear analysis of (2.1)-(2.9) in the limit (2.12) involves only solutions that are close to the static steady state $u \equiv w \equiv q-1 \equiv 0, f \equiv 1$. Since the capillary Reynolds number $C^{-1}$ is large, we are led to consider four distinguished regions in the liquid bridge (see figure 1): (a) two Stokes boundary layers near the disks; $(b)$ an interface boundary layer near the free surface; $(c)$ two corner tori near the border of the disks; and $(d)$ the bulk, that is the remaining part of the liquid bridge. The characteristic size of regions $(a),(b)$ and $(c)$ (where inertia and viscous terms in momentum conservation equations are comparable) is readily seen to be of order $C^{1 / 2}$.

† In order to emphasize the main ideas in the derivation below, some involved expressions have been relegated to an Appendix, and for the sake of brevity some other intermediate expressions have been omitted. A more detailed derivation may be obtained from the JFM editorial office or from the authors. 
The solution in the bulk is written as

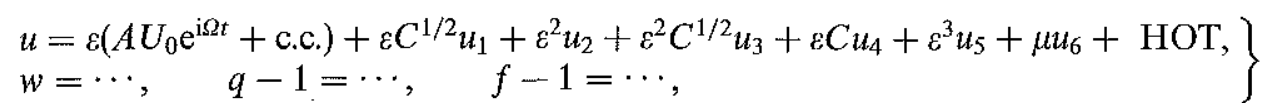

where the expansions for $w, q-1$ and $f-1$ are similar to that for $u$ and hereafter HOT and c.c. stand for higher-order terms than those displayed and the complex conjugate respectively. $\left(U_{0}, W_{0}, Q_{0}, F_{0}\right)$ is a non-trivial eigenmode of the inviscid linear problem, $\Omega \neq 0$ is the associated eigenfrequency, $A$ is the complex amplitude, which depends weakly on time (i.e. $|\mathrm{d} A / \mathrm{d} t| \ll|A|$ ), and the real parameter $\varepsilon$ satisfies

$$
0<\varepsilon \ll 1 \text {. }
$$

In order to define the complex amplitude $A$ we must impose an additional condition at each asymptotic order. If we impose

$$
\int_{0}^{2 \pi / \Omega} \int_{-A}^{\Lambda} \int_{0}^{1}\left(U_{0} u_{k}+W_{0} w_{k}\right) \mathrm{e}^{-\mathrm{i} \Omega t} r \mathrm{~d} r \mathrm{~d} z \mathrm{~d} t=0 \quad \text { for all } k \geqslant 1,
$$

then $A$ is defined as $\varepsilon A=\Omega \iiint\left(U_{0} u+W_{0} w\right) \mathrm{e}^{-\mathrm{i} \Omega t} r \mathrm{~d} r \mathrm{~d} z \mathrm{~d} t\left[2 \pi \iint\left(U_{0}^{2}+W_{0}^{2}\right) r \mathrm{~d} r \mathrm{~d} z\right]^{-1}$.

The main goal in this section is to derive the following evolution equation for the complex amplitude:

$$
\varepsilon \mathrm{d} A / \mathrm{d} t=-\left[(1+\mathrm{i}) \alpha_{1} C^{1 / 2}+\alpha_{2} C\right] \varepsilon A+\mathrm{i} \alpha_{3} \varepsilon^{3} A|A|^{2}+\mathrm{i} \mu\left(\alpha_{4}^{+} \beta_{+} \mathrm{e}^{\mathrm{i} \omega_{+} \delta t}-\alpha_{4}^{-} \beta_{-} \mathrm{e}^{\mathrm{i} \omega \omega_{-} \delta t}\right)+\text { HOT, }
$$

where $\alpha_{1}, \alpha_{2}, \alpha_{3}$ and $\alpha_{4}^{ \pm}$are real constants that will be calculated below $\left(\alpha_{1}\right.$ and $\alpha_{2}$ have already been calculated by Higuera et al. 1994).

The well-known cubic amplitude equation ( $3.3 a$ ) is a balance between inertia, damping, nonlinearity and forcing. Its specific feature here is that we are using a two-terms approximation for the damping rate, $\alpha_{1} C^{1 / 2}+\alpha_{2} C$, because $\alpha_{1}$ is about $10^{-2}$ times $\alpha_{2}$ (see figures 2 and 4 below). That approximation of the damping rate is quite good for moderately small values of $C$ (roughly, $C \leqslant 0.01$ ), while if the second term were ignored, then the resulting approximation would be useful only for extremely small values of $C$ (roughly, $C \leqslant 10^{-6}$, see Higuera et al. 1994). Notice that we are not introducing particular scalings relating the small parameters $C, \mu, \delta$ and $\varepsilon$, and the characteristic size of the slower time scale for the evolution of the complex amplitude $A$. This point will be discussed in $\S 4$, when analysing $(3.3 a)$.

In order to obtain the amplitude equation $(3.3 a)$ we could proceed in a straightforward manner, as follows. We would substitute (3.1) into (2.1)-(2.3) to obtain a recursive system of equations for $\left(u_{1}, w_{1}, q_{1}, f_{1}\right), \ldots$, in the bulk; the boundary conditions to be applied to those equations would be obtained by means of the appropriate matching conditions with the solutions in the boundary layers. Finally, the evolution equation for $A$ would be obtained by applying the appropriate solvability conditions (i.e. by eliminating secular terms in the time scale $t \sim 1$ ) to the equations in the bulk at each asymptotic order. But then we would encounter two main difficulties. First, we would need to derive equations and boundary conditions for the seven terms displayed in (3.1). This would make calculations quite tedious (the solutions in the interface boundary layer and the associated matching conditions are especially involved). Secondly, the solution in the bulk exhibits a singularity, near the border of the disks, that is more and more important as one proceeds with higher-order terms in the expansions (3.1); the singularity must be handled carefully at order $\varepsilon C$ (and perhaps at orders $\varepsilon^{2} C^{1 / 2}$ and $\varepsilon^{3}$ ) to avoid wrong results when applying solvability 
conditions. This difficulty, first encountered by Ursell (1952), is always present at the interaction of interfaces and solid boundaries. We shall avoid both difficulties by deriving in $\$ 3.4$ an appropriate integral solvability condition for (2.1)-(2.9) that is equivalent to the set of solvability conditions (at orders $\varepsilon C^{1 / 2}, \varepsilon^{2}, \ldots$ ) mentioned above, but depends only on the first three terms in (3.1), that are first considered below.

The amplitude equation is assumed to be of the type

$$
\delta \varepsilon \mathrm{d} A / \mathrm{d} \tau=H_{1} \varepsilon C^{1 / 2}+H_{2} \varepsilon^{2}+H_{3} \varepsilon C+H_{4} \varepsilon^{2} C^{1 / 2}+H_{5} \varepsilon^{3}+H_{6} \mu+\text { HOT, }
$$

where the complex coefficients $H_{1}, \ldots, H_{6}$ are to be calculated and the slow time variable $\tau$ is defined as

$$
\tau=\delta t .
$$

Thus we are considering two time scales: $t \sim 1$ that is a capillary time scale and $\tau \sim 1$ that (when appropriately selecting the small parameters) will be the time scale associated with both damping of capillary oscillations (in \$4) and the streaming flow (in $\S 5$ ).

\subsection{The solution in the bulk}

As mentioned above, we shall only need to calculate the first three terms in the expansions (3.1). The (linear) inviscid eigenmode $\left(U_{0}, W_{0}, Q_{0}, F_{0}\right)$ is given by

$$
\begin{aligned}
& U_{0 r}+r^{-1} U_{0}+W_{0 z}=\mathrm{i} \Omega U_{0}+Q_{0 r}=\mathrm{i} \Omega W_{0}+Q_{0 z}=0, \\
& W_{0}=0 \quad \text { at } z= \pm \Lambda, \quad U_{0}=W_{0 r}=0 \quad \text { at } r=0, \\
& U_{0}-\mathrm{i} \Omega F_{0}=Q_{0}+F_{0}+F_{0}^{\prime \prime}=0 \quad \text { at } r=1, \\
& F_{0}( \pm \Lambda)=\int_{-\Lambda}^{\Lambda} F_{0}(z) \mathrm{d} z=0 .
\end{aligned}
$$

The terms of orders $\varepsilon C^{1 / 2}$ and $\varepsilon^{2}$ in (3.1) are given by

$$
\begin{aligned}
& u_{k r}+r^{-1} u_{k}+w_{k z}=0, \\
& u_{k t}+q_{k r}+\left(H_{k} U_{0} \mathrm{e}^{\mathrm{i} \Omega t}+\text { c.c. }\right)=w_{k t}+q_{k z}+\left(H_{k} W_{0} \mathrm{e}^{\mathrm{i} \Omega t}+\text { c.c. }\right)=0, \\
& w_{k}=G_{k}^{ \pm} \quad \text { at } z= \pm A, \quad u_{k}=w_{k r}=q_{k r}=0 \quad \text { at } r=0, \\
& u_{k}=\phi_{k}, \quad q_{k}=\psi_{k} \quad \text { at } r=1, \\
& f_{k}=0 \quad \text { at } z= \pm \Lambda, \quad \int_{-A}^{A} f_{k} \mathrm{~d} z+\gamma_{k}=0,
\end{aligned}
$$

for $k=1$ and 2, where

$$
\gamma_{1}=0, \quad \gamma_{2}=\int_{-A}^{\Lambda}\left(A^{2} F_{0}^{2} \mathrm{e}^{\mathrm{i} \Omega t}+\text { c.c. }+2\left|A F_{0}\right|^{2}\right) \mathrm{d} z / 2 .
$$

Equations (3.9), (3.10) and (3.13) and the boundary conditions at $r=0$ are obtained upon substitution of (3.1) and (3.3b) into (2.1)-(2.3), (2.5) and (2.10), when taking into account that $U_{0 z} \equiv W_{0 r}$. The remaining boundary conditions (and the functions $G_{k}^{ \pm}$, $\phi_{k}$ and $\psi_{k}$ ) will be obtained below by applying matching conditions with the Stokes and the interface boundary layers.

\subsection{The solution in the Stokes boundary layers}

For the sake of brevity we shall give (only some) details for the boundary layer near $z=\Lambda$, where we use the stretched coordinate

$$
\xi=\left[z-\Lambda-h_{+}(t)\right] / C^{1 / 2},
$$


with $h_{+}$as given in (2.11). We seek the expansions

$$
\left.\begin{array}{rl}
u & =\varepsilon A \widetilde{U}_{0} \mathrm{e}^{\mathrm{i} \Omega t}+\text { c.c. }+\varepsilon C^{1 / 2} \tilde{u}_{1}+\varepsilon^{2} \tilde{u}_{2}+\text { HOT, } \\
w & =h_{+}^{\prime}+C^{1 / 2}\left(\varepsilon A \widetilde{W}_{0} \mathrm{e}^{\mathrm{i} \Omega t}+\text { c.c. }+ \text { HOT }\right), \\
-1 & =\varepsilon A \widetilde{Q}_{0} \mathrm{e}^{\mathrm{i} \Omega t}+\text { c.c. }+\varepsilon C^{1 / 2} \tilde{q}_{1}+\varepsilon^{2} \tilde{q}_{2}+\text { HOT }+O(\mu) .
\end{array}\right\}
$$

Substitution of (3.15) and (3.3b) into (2.1)-(2.4) provides a system of recursive equations and boundary conditions, whose solution yields

$$
\begin{aligned}
\widetilde{U}_{0}= & K_{0}^{+}(r)(1-\Gamma), \\
\widetilde{W}_{0}= & -\left(\mathrm{d} K_{0}^{+} / \mathrm{d} r+r^{-1} K_{0}^{+}\right)\left[\xi+(1-\mathrm{i})(1-\Gamma) /(2 \Omega)^{1 / 2}\right], \\
\tilde{u}_{1}= & {\left[K_{1}^{+}(1-\Gamma)-(1-\mathrm{i}) H_{1} K_{0}^{+} \xi \Gamma / 2(2 \Omega)^{1 / 2}\right] A \mathrm{e}^{\mathrm{i} \Omega t}+\text { c.c. }, } \\
\tilde{u}_{2}= & |A|^{2} \bar{K}_{0}^{+}\left(\mathrm{d} K_{0}^{+} / \mathrm{d} r+r^{-1} K_{0}^{+}\right) \\
& \times\left[\left[\mathrm{i}\left(|\Gamma|^{2}-1\right)+2(1-2 \mathrm{i})(\bar{\Gamma}-1)+(1+\mathrm{i})(2 \Omega)^{1 / 2} \xi \bar{\Gamma}\right] / 2 \Omega\right. \\
& +|A|^{2} \bar{K}_{0}^{+}\left(\mathrm{d} K_{0}^{+} / \mathrm{d} r\right)\left[|\Gamma|^{2}-1+2 \mathrm{i}(\Gamma-\bar{\Gamma})\right] / 2 \Omega+\text { c.c. }+ \text { ROT, }
\end{aligned}
$$

where overbars and c.c. stand for the complex conjugate, ROT stand for rapidly oscillatory terms, depending on the fast time variable as $\exp (\mathrm{i} m \Omega t)$, with $m=$ non-zero integer, $K_{0}^{+}$and $K_{1}^{+}$are arbitrary functions of $r$ (that are to be calculated) and $\Gamma=\Gamma(\xi)$ is given by

$$
\Gamma(\xi)=\exp \left[(1+\mathrm{i})(\Omega / 2)^{1 / 2} \xi\right] .
$$

Now, the functions $K_{0}^{+}, K_{1}^{+}$and the functions $G_{1}^{+}$and $G_{2}^{+}$appearing in the boundary conditions (3.11) are obtained by applying matching conditions between the solutions in the bulk (3.1) and in this boundary layer (3.15). After applying a similar procedure to the boundary layer near $z=-\Lambda$ we obtain

$$
\begin{aligned}
K_{0}^{ \pm}(r) & =U_{0}(r, \pm \Lambda), \quad u_{1}(r, \pm \Lambda)=A K_{1}^{ \pm}(r) \mathrm{e}^{\mathrm{i} \Omega t}+\text { c.c. } \\
G_{1}^{ \pm} & = \pm\left[(1-\mathrm{i}) A W_{0 z}(r, \pm \Lambda) \mathrm{e}^{\mathrm{i} \Omega t}+\text { c.c. }\right] /(2 \Omega)^{1 / 2}, \quad G_{2}^{ \pm}=0, \\
u_{2} & =-|A|^{2}\left[3(1-\mathrm{i}) \bar{U}_{0} U_{0 r}+\text { c.c. }+4 r^{-1}\left|U_{0}\right|^{2}\right] / 2 \Omega+\text { ROT, } \quad \text { at } z= \pm \Lambda,
\end{aligned}
$$

where the first continuity equation (3.5) has been taken into account and $u_{2}$ has been also obtained for convenience.

\subsection{The solution in the interface boundary layer}

Here we use the following stretched coordinate and seek the following expansions:

$$
\begin{gathered}
\eta=[r-f(z, t, \tau)] / C^{1 / 2}, \\
u=f_{t}+\delta f_{\tau}+f_{z} w+C^{1 / 2}\left(\varepsilon A U_{0}^{*} \mathrm{e}^{\mathrm{i} \Omega t}+\text { c.c. }+\varepsilon C^{1 / 2} u_{1}^{*}+\text { HOT }\right), \\
w=\varepsilon A W_{0}^{*} \mathrm{e}^{\mathrm{i} \Omega t}+\text { c.c. }+\varepsilon C^{1 / 2} w_{1}^{*}+\varepsilon^{2} w_{2}^{*}+\varepsilon^{2} C^{1 / 2} w_{3}^{*}+\text { HOT } \\
q-1=\left(u^{2}+w^{2}\right) / 2+\left(\varepsilon A P_{0}^{*} \mathrm{e}^{\mathrm{i} \Omega t}+\text { c.c. }\right)+\varepsilon C^{1 / 2} p_{1}^{*}+\varepsilon^{2} p_{2}^{*}+\varepsilon^{2} C^{1 / 2} p_{3}^{*}+\text { HOT. }
\end{gathered}
$$

Substitution of (3.23)-(3.24), the last expansion (3.1) and (3.3b) into (2.1)-(2.3) and (2.6)-(2.8) provides a system of recursive equations and boundary conditions whose 
solution yields

$$
\left.\begin{array}{rl}
P_{0}^{*}= & -\left(F_{0}+F_{0}^{\prime \prime}\right), \quad W_{0}^{*}=-\mathrm{i}\left(F_{0}^{\prime}+F_{0}^{\prime \prime \prime}\right) / \Omega, \quad U_{0}^{*}=\mathrm{i}\left(F_{0}^{\prime \prime}+F_{0}^{\mathrm{iv}}-\Omega^{2} F_{0}\right) \eta / \Omega, \\
w_{1}^{*}= & -(2 \Omega)^{1 / 2}\left[(1+\mathrm{i}) A F_{0}^{\prime} \Gamma(\eta) \mathrm{e}^{\mathrm{i} \Omega t}+\text { c.c. }\right]+\left(\mathrm{i} \Omega A F_{0}^{\prime} \mathrm{e}^{\mathrm{i} \Omega t}+\text { c.c. }\right) \eta+\text { POL }, \\
p_{1}^{*}= & -f_{1}-f_{1 z z}+\Omega^{2}\left(A F_{0} \mathrm{e}^{\mathrm{i} \Omega t}+\text { c.c. }\right) \eta, \quad u_{1}^{*}=2\left[A F_{0}^{\prime \prime} \Gamma(\eta) \mathrm{e}^{\mathrm{i} \Omega t}+\text { c.c. }\right]+\text { POL, } \\
p_{2}^{*}= & -f_{2}-f_{2 z z}+\left(A F_{0} \mathrm{e}^{\mathrm{i} \Omega t}+\text { c.c. }\right)^{2}-\left(A F_{0}^{\prime} \mathrm{e}^{\mathrm{i} \Omega t}+\text { c.c. }\right)^{2} / 2, \quad w_{2 \eta}^{*}=0, \quad p_{3}^{*}=\text { POL, } \\
w_{3 \eta}^{*}=\mathrm{i}|A|^{2}\left[\left(2 \bar{F}_{0}^{\mathrm{iv}}+2 \bar{F}_{0}^{\prime \prime}-\Omega^{2} \bar{F}_{0}\right) F_{0}^{\prime}(3-2 \Gamma(\eta))+\left(\bar{F}_{0}^{\prime \prime \prime}+\bar{F}_{0}^{\prime}\right) F_{0}^{\prime \prime}(1-2 \Gamma(\eta))\right] / \Omega \\
& -(1-\mathrm{i})(2 / \Omega)^{1 / 2}|A|^{2}\left(\bar{F}_{0}^{\mathrm{iv}}+\bar{F}_{0}^{\prime \prime}-\Omega^{2} \bar{F}_{0}\right) F_{0}^{\prime} \eta \Gamma(\eta)+\text { c.c. }+\mathrm{ROT},
\end{array}\right\}
$$

where the function $\Gamma$ is as defined in (3.19), POL stands for a polynomial in the $\eta$ variable (whose coefficients may depend on the remaining variables) and, as above, ROT stands for rapidly oscillatory terms. Now, in order to apply matching conditions between the solution in the bulk (3.1) and the solution in this boundary layer (3.24), we take into account that the solution in the bulk satisfies

$$
\begin{gathered}
q(f, z ; t, \tau)=q(1, z ; t, \tau)+(f-1) q_{r}(1, z ; t, \tau)+O\left(\varepsilon^{3}\right), \\
u(f, z ; t, \tau)=\ldots, \quad w_{r}(f, z ; t, \tau)=\ldots,
\end{gathered}
$$

to obtain, at $r=1$,

$$
\begin{aligned}
\phi_{1} \equiv u_{1}= & f_{1 t}+\left(H_{1} F_{0} \mathrm{e}^{\mathrm{i} \Omega t}+\text { c.c. }\right), \quad \psi_{1} \equiv q_{1}=-f_{1}-f_{1 z z} \\
\phi_{2} \equiv u_{2}= & f_{2 t}+\left(H_{2} F_{0} \mathrm{e}^{\mathrm{i} \Omega t}+\text { c.c. }\right)-\left(A F_{0} \mathrm{e}^{\mathrm{i} \Omega t}+\text { c.c. }\right)\left(A U_{0 r} \mathrm{e}^{\mathrm{i} \Omega t}+\text { c.c. }\right) \\
& +\left(A F_{0}^{\prime} \mathrm{e}^{\mathrm{i} \Omega t}+\text { c.c. }\right)\left(A W_{0} \mathrm{e}^{\mathrm{i} \Omega t}+\text { c.c. }\right) \\
\psi_{2} \equiv q_{2}= & -f_{2}-f_{2 z z}-\left(A F_{0} \mathrm{e}^{\mathrm{i} \Omega t}+\text { c.c. }\right)\left(A Q_{0 r} \mathrm{e}^{\mathrm{i} \Omega t}+\text { c.c. }\right)+\left(A F_{0} \mathrm{e}^{\mathrm{i} \Omega t}+\text { c.c. }\right)^{2} \\
& -\left(A F_{0}^{\prime} \mathrm{e}^{\mathrm{i} \Omega t}+\text { c.c. }\right)^{2} / 2+\left(A W_{0} \mathrm{e}^{\mathrm{i} \Omega t}+\text { c.c. }\right)^{2} / 2+\left(i \Omega A F_{0} \mathrm{e}^{\mathrm{i} \Omega t}+\text { c.c. }\right)^{2} / 2 \\
w_{2 r}= & \mathrm{i}|A|^{2}\left[3\left(2 \bar{F}_{0}^{\mathrm{iv}}+2 \bar{F}_{0}^{\prime \prime}-\Omega^{2} \bar{F}_{0}\right) F_{0}^{\prime}+\left(\bar{F}_{0}^{\prime \prime \prime}+\bar{F}_{0}^{\prime}\right) F_{0}^{\prime \prime}\right] / \Omega \\
& -|A|^{2} \bar{W}_{0 r r} F_{0}+\text { c.c. }+ \text { ROT. }
\end{aligned}
$$

\subsection{Solvability conditions}

Here we shall calculate the coefficients in the amplitude equation $(3.3 b)$ by eliminating secular terms in the short time scale, $t \sim 1$, that is, by requiring the solution of (2.1)(2.8) to be bounded as $t \rightarrow \infty$ (for each fixed value of $\tau$ ). To this end, we first obtain an integral solvability condition as follows. First, introduce into (2.1)-(2.8) and (2.11) the time scales $t \sim 1$ and $\tau=\delta t$ by replacing in (2.2)-(2.4) and (2.6) the time derivative by $\partial / \partial t+\delta \partial / \partial \tau$, and in (2.11), $\mathrm{i}\left(\Omega+\omega_{ \pm} \delta\right) t$ by $\mathrm{i}\left(\Omega t+\omega_{ \pm} \tau\right)$. Then multiply (2.2) by $r U_{0} \mathrm{e}^{-\mathrm{i} \Omega t},(2.3)$ by $r W_{0} \mathrm{e}^{-\mathrm{i} \Omega t}$, the second and third equations (3.5) by $-r u \mathrm{e}^{-\mathrm{i} \Omega t}$ and $-r w \mathrm{e}^{-\mathrm{i} \Omega t}$ respectively, add, integrate in $0<r<f,-\Lambda+h_{-}<z<A+h_{+}$, integrate by parts and take into account the boundary conditions (2.4) and (2.6)-(2.9) to obtain

$$
\frac{\partial}{\partial t}\left(\mathrm{e}^{-\mathrm{i} \Omega t} I_{1}\right)+\mathrm{e}^{-\mathrm{i} \Omega t} I_{2}=\mathrm{e}^{-\mathrm{i} \Omega t}\left(I_{3}+I_{4}+I_{5}^{+}-I_{5}^{-}\right),
$$

where

$$
\begin{aligned}
& I_{1}=\int_{-A+h_{-}}^{\Lambda+h_{+}} \int_{0}^{f}\left(u U_{0}+w W_{0}\right) r \mathrm{~d} r \mathrm{~d} z-\int_{-\Lambda+h_{-}}^{\Lambda+h_{+}} Q_{0}(f, z) f(f-1) \mathrm{d} z, \\
& I_{2}=\int_{-\Lambda+h_{-}}^{\Lambda+h_{+}} \int_{0}^{f} \delta\left(u_{\tau} U_{0}+w_{\tau} W_{0}\right) r \mathrm{~d} r \mathrm{~d} z-\int_{-\Lambda+h_{-}}^{\Lambda+h_{+}} \delta f_{\tau} f Q_{0}(f, z) \mathrm{d} z,
\end{aligned}
$$




$$
\begin{aligned}
& I_{3}=\int_{-A+h_{-}}^{A+h_{+}} \int_{0}^{f}\left(u W_{0}-w U_{0}\right)\left(u_{z}-w_{r}\right) r \mathrm{~d} r \mathrm{~d} z \\
& -C \int_{-A+h_{-}}^{\Lambda+h_{+}} \int_{0}^{f}\left[\left(U_{0 r} u_{r}+U_{0 z} u_{z}+W_{0 r} w_{r}+W_{0 z} w_{z}\right) r+U_{0} u / r\right] \mathrm{d} r \mathrm{~d} z, \\
& \left.I_{4}=\int_{-\Lambda+h_{-}}^{\Lambda+h_{+}}\left[\left(u U_{0}+w W_{0}\right) f-f(f-1)\right) Q_{0 r}-(f-1) Q_{0}\right]_{r=f} f_{t} \mathrm{~d} z \\
& +\int_{-\Lambda+h_{-}}^{\Lambda+h_{+}}\left[\mathrm{i} \Omega(f-1) Q_{0}+\left(U_{0}-f_{z} W_{0}\right)\left(1+\frac{f f_{z z}-1-f_{z}^{2}}{f\left(1+f_{z}^{2}\right)^{3 / 2}}-\frac{u^{2}+w^{2}}{2}\right)\right. \\
& \left.+C U_{0}\left(u_{r}-f_{z} u_{z}\right)\right]_{r=f} f \mathrm{~d} z-C \int_{-\Lambda+h_{-}}^{\Lambda+h_{*} \cdot}\left[W_{0}\left(u_{z}+f_{z} w_{z}+2 f_{z} \frac{u_{r}-w_{z}}{1-f_{z}^{2}}\right)\right. \\
& \left.+2\left(U_{0}-f_{z} W_{0}\right) \frac{u_{r}-\left(w_{r}+u_{z}\right) f_{z}+w_{z} f_{z}^{2}}{1+f_{z}^{2}}\right]_{r=f} f \mathrm{~d} z \\
& I_{5}^{ \pm}=\int_{0}^{1}\left[\left(\frac{\partial h_{ \pm}}{\partial t} W_{0}+Q_{0}\right) \frac{\partial h_{ \pm}}{\partial t}+\delta Q_{0} \frac{\partial h_{ \pm}}{\partial \tau}-W_{0}(q-1)+C\left(U_{0} u_{z}+W_{0} w_{z}\right)\right]_{z= \pm \lambda+h_{ \pm}} r \mathrm{~d} r .
\end{aligned}
$$

Now, secular terms are eliminated by integrating (3.30) in the short time variable $t$, dividing by $t$, letting $t \rightarrow \infty$ and requiring $I_{1}$ to be bounded as $t \rightarrow \infty$. Then we obtain the following integral solvability condition:

$$
\lim _{t \rightarrow \infty} t^{-1} \int_{0}^{t} \mathrm{e}^{-\mathrm{i} \Omega t}\left(I_{2}-I_{3}-I_{4}-I_{5}^{+}+I_{5}^{-}\right) \mathrm{d} t=0
$$

or

$$
\text { resonant part of }\left(I_{2}-I_{3}-I_{4}-I_{5}^{+}+I_{5}^{-}\right)=0 \text {, }
$$

where by resonant part we mean that part depending on the short time variable precisely as $\exp (\mathrm{i} \Omega t)$, and we have taken into account that the remaining, nonresonant part of $\left(I_{2}-I_{3} \cdots\right)$ (that is, those terms depending on $t$ as $\exp (\mathrm{i} m \Omega t)$, with $m \neq 1$ being an integer) does not contribute to the limit in (3.31a).

Before proceeding further, let us briefly discuss the integral solvability condition (3.31b), which exhibits two advantages (that are related to each other): the coefficients of the amplitude equation ( $3.3 \mathrm{~b}$ ) will be obtained below (i) without the need of explicitly considering the terms of orders $\varepsilon C, \varepsilon^{2} C^{1 / 2}$ and $\varepsilon^{3}$ in the expansions (3.1) and (ii) without the need of analysing the corner region (c) (see figure 1) to avoid wrong results (as usually done, see, e.g., Mei \& Liu 1973). This will be so because (3.31b) may be considered (but only to some extent) to be the result of adding up the solvability conditions for the problems giving the five last terms in the expansions (3.1). As mentioned above, these five terms exhibit singularities near the corner region (that become stronger and stronger as one proceeds in the perturbation process) that make an integration by parts step fail (at least ar order $\varepsilon C$ ) in the process of deriving the associated solvability condition. But all manipulations leading to $(3.31 \mathrm{~b})$ were done with the exact solution of (2.1)-(2.8) in the exact fluid domain $0<r<f$, $-\Lambda+h_{-}<z<A+h_{+}$; since the exact solution exhibits only a very weak singularity at the borders of the disks, $r=1, z= \pm A+h_{ \pm}$, all manipulations above (including integration by parts) leading to (3.30) were correct, and the danger of wrong results was avoided. Condition $(3.31 \mathrm{~b}$ ) may be also seen as an alternative to the integral equation (obtained from the mechanical energy conservation equation) that was used by Ursell (1952) to obtain (without surprises) the damping rate (i.e. the real part 
of the coefficient of $-A$ in the right-hand side of $(3.3 b)$ ) in a related problem; our integral equation also provides the imaginary part of the coefficient of $A$ and the remaining coefficients in $(3.3 b)$.

In order to apply the solvability conditions $(3.31 b)$ it is first convenient to take into account (2.6), (2.11) and (3.5)-(3.7), and the structure of the solution in the bulk and in the Stokes and the interface boundary layers to re-write the expressions for $I_{2}, \ldots, I_{5}^{ \pm}$above as given in (A1)-(A4) in the Appendix. Now, the coefficients $H_{1}, H_{2}$ and $H_{6}$ of the amplitude equation (3.3b) are readily calculated upon substitution of (3.1) and (3.3b) into (3.31b) and setting to zero the coefficients of $\varepsilon C^{1 / 2}, \varepsilon^{2}$ and $\mu$, to obtain

$$
H_{1}=-(1+\mathrm{i}) \alpha_{1} A, \quad H_{2}=0, \quad H_{6}=\mathrm{i}\left(\alpha_{4}^{+} \beta_{+} \mathrm{e}^{\mathrm{i} \omega_{+} \tau}-\alpha_{4}^{-} \beta_{-} \mathrm{e}^{\mathrm{i} \omega-\tau}\right),
$$

where

$$
\begin{aligned}
& \alpha_{1}=\int_{0}^{1} Q_{0 r}(r, \Lambda)^{2} r \mathrm{~d} r\left[\left(2 \Omega^{3}\right)^{1 / 2} \int_{-\Lambda}^{\Lambda} F_{0}(z) Q_{0}(1, z) \mathrm{d} z\right]^{-1}, \\
& \alpha_{4}^{ \pm}=-\Omega \int_{0}^{1} Q_{0}(r, \Lambda) r \mathrm{~d} r\left[2 \int_{-\Lambda}^{\Lambda} F_{0}(z) Q_{0}(1, z) \mathrm{d} z\right]^{-1},
\end{aligned}
$$

and we have taken into account the equation

$$
\int_{-\Lambda}^{\Lambda} \int_{0}^{1}\left(U_{0}^{2}+W_{0}^{2}\right) r \mathrm{~d} r \mathrm{~d} z=-\int_{-\Lambda}^{\Lambda} F_{0}(z) Q_{0}(1, z) \mathrm{d} z,
$$

that is readily obtained when multiplying the second and third equations of (3.5) by $r U_{0}$ and $r W_{0}$ respectively, adding, integrating, integrating by parts and taking into account (3.5)-(3.7).

The constants $\alpha_{1}$ and $\alpha_{4}^{\ddagger}$ are readily calculated when using the expressions for $Q_{0}$ and $F_{0}$ in (A9a) or (A9b) (in the Appendix). Notice that these constants are real and that

$$
\alpha_{4}^{-}=-\alpha_{4}^{+} \text {for odd modes, and } \alpha_{4}^{-}=\alpha_{4}^{+} \text {for even modes. }
$$

The constants $\alpha_{1}$ and $\alpha_{4}^{ \pm}$are plotted in terms of $\Lambda$ in figures 2 and 3; for the first capillary mode, $\alpha_{4}^{+}$diverges at the capillary instability limit, $\Lambda=\pi$, where our analysis breaks down, as will be remarked in $\S 3.5$.

The coefficients $\mathrm{H}_{3}, \mathrm{H}_{4}$ and $\mathrm{H}_{5}$ in the amplitude equation (3.3b) will depend on the terms of orders $\varepsilon C^{1 / 2}$ and $\varepsilon^{2}$ in the expansions (3.1), which are considered now. When taking into account (3.14), (3.21), (3.26)-(3.28) and (3.32), the solutions of (3.2b), (3.5)-(3.13) for $k=1$ and 2 are seen to be as given by

$$
\begin{aligned}
u_{1}= & A U_{1} \mathrm{e}^{\mathrm{i} \Omega t}+\text { c.c., } \quad w_{1}=A W_{1} \mathrm{e}^{\mathrm{i} \Omega t}+\text { c.c. } \\
& q_{1}=A Q_{1} \mathrm{e}^{\mathrm{i} \Omega t}+\text { c.c. }, \quad f_{1}=A F_{1} \mathrm{e}^{\mathrm{i} \Omega t}+\text { c.c. }, \\
u_{2}= & A^{2} U_{22} \mathrm{e}^{2 i \Omega t}+\text { c.c. }+u_{20}, \quad w_{2}=A^{2} W_{22} \mathrm{e}^{2 i \Omega t}+\text { c.c. }+w_{20}, \\
q_{2}= & A^{2} Q_{22} \mathrm{e}^{2 i \Omega t}+\text { c.c. }+|A|^{2} Q_{20}, \quad f_{2}=A^{2} F_{22} \mathrm{e}^{2 i \Omega t}+\text { c.c. }+|A|^{2} F_{20},
\end{aligned}
$$

where the non-oscillatory (in the short time scale) components of the velocity field, $u_{20}$ and $w_{20}$, will be considered in $\S 5$, while $\left(U_{1}, W_{1}, Q_{1}, F_{1}\right),\left(U_{22}, W_{22}, Q_{22}, F_{22}\right)$ and $\left(Q_{20}, F_{20}\right)$ are as given by (A10)-(A19), in the Appendix. Notice that the problem (3.5)-(3.13) for $k=1$ is solvable precisely because $H_{1}$ is as given in (3.32), and that the problem (3.5)-(3.13) has a solution for $k=2$ only if neither $\Omega_{1}=2 \Omega$ nor $\Omega_{1}=0$ is an inviscid eigenfrequency associated with an even mode (i.e. satisfies the second equation of (A6)). 


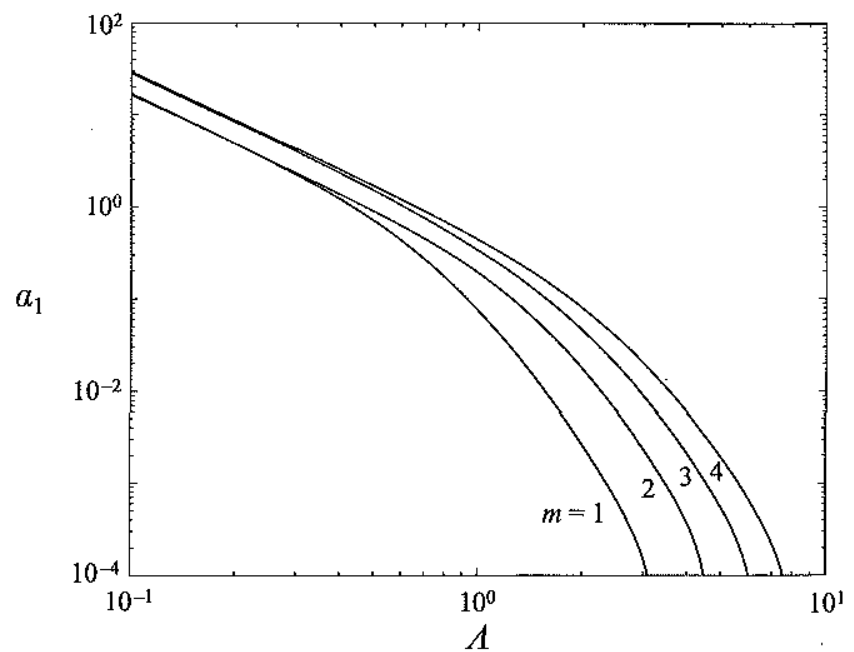

FIGURE 2. The coefficient $\alpha_{1}$ in the leading term of the damping rate for the first four modes.

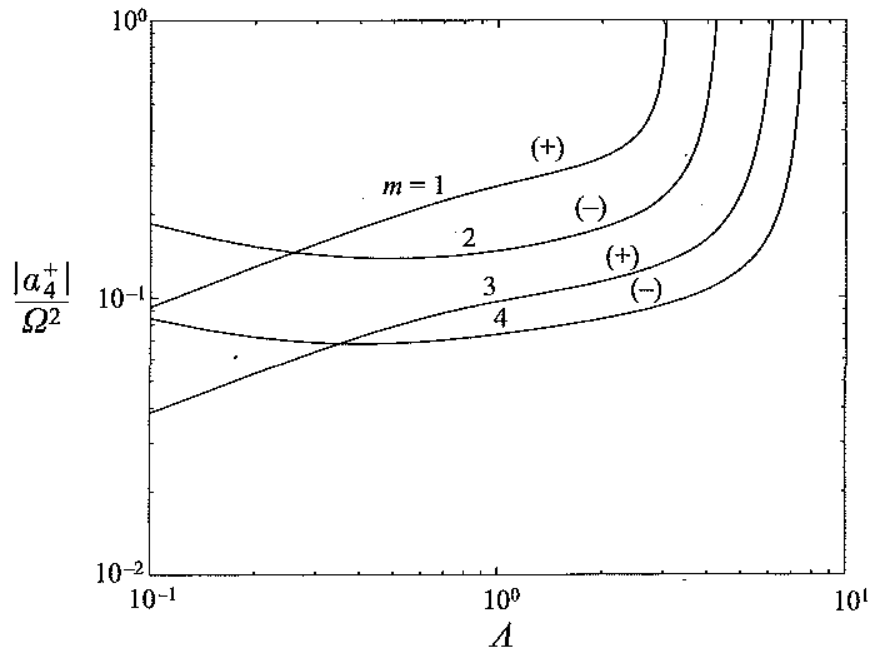

Figure 3. The coefficient $\alpha_{4}^{+} / \Omega^{2}$ in the forcing term of the amplitude equation (3.3a) for the first four modes. The sign of $\alpha_{4}^{+}$is indicated.

Now $\mathrm{H}_{3}, \mathrm{H}_{4}$ and $\mathrm{H}_{5}$ are calculated upon substitution of (3.1) and (3.3b) into (3.31b) (with $I_{2}, \ldots, I_{5}^{ \pm}$as given in (A1)-(A4), in the Appendix) and setting to zero the coefficients of $\varepsilon C, \varepsilon^{2} C^{1 / 2}$ and $\varepsilon^{3}$, to obtain (after some tedious algebraic manipulations)

$$
H_{3}=-\alpha_{2} A, \quad H_{4}=0, \quad H_{5}=\mathrm{i} \alpha_{3} A|A|^{2},
$$

where the real constants $\alpha_{2}$ and $\alpha_{3}$ are given by

$$
\begin{aligned}
\left(\alpha_{2}-\right. & \left.2-2 \alpha_{1}^{2} / \Omega\right) \int_{-\Lambda}^{\Lambda} F_{0}(z) Q_{0}(1, z) \mathrm{d} z=(1+\mathrm{i}) \int_{0}^{1} Q_{0 r}(r, \Lambda) Q_{1 r}(r, \Lambda) r \mathrm{~d} r /\left(2 \Omega^{3}\right)^{1 / 2} \\
& +\int_{-\Lambda}^{\Lambda}\left[2 Q_{0}(1, z)^{2}-\Omega^{2} F_{0}(z)^{2}-(1+\mathrm{i}) \alpha_{1} F_{1}(z) Q_{0}(1, z)\right] \mathrm{d} z-4 F_{0}^{\prime}(\Lambda) F_{0}^{\prime \prime}(\Lambda),
\end{aligned}
$$




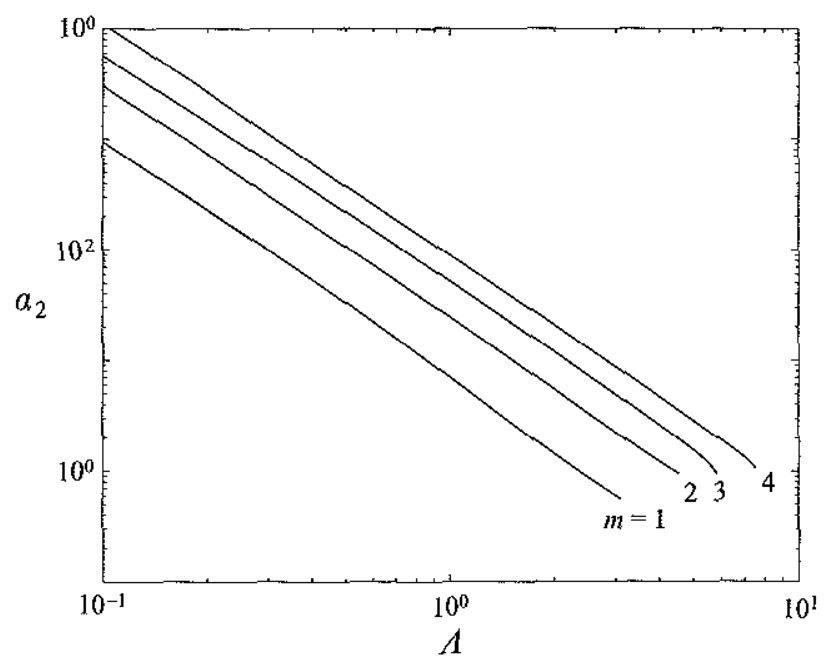

FrGURE 4. The coefficient $\alpha_{2}$ in the first correction to the damping rate for the first four modes.

$$
\begin{gathered}
2\left(\alpha_{3} / \Omega\right) \int_{-A}^{\Lambda} F_{0}(z) Q_{0}(1, z) \mathrm{d} z=\int_{-A}^{\Lambda}\left[\left(2-3 \Omega^{2}\right) F_{0} F_{22}-F_{0}^{\prime} F_{22}^{\prime}+\left(2-\Omega^{2}\right) F_{0} F_{20}-F_{0}^{\prime} F_{20}^{\prime}\right. \\
\left.-2 W_{0}(1, z) W_{22}(1, z)-\left(F_{22}+F_{20}\right) Q_{0}(1, z)\right] F_{0} \mathrm{~d} z+\int_{-A}^{\Lambda}\left(F_{22}-F_{20}\right) W_{0}(1, z)^{2} \mathrm{~d} z \\
+\int_{-\Lambda}^{A}\left[\left(11 F_{0}+17 F_{0}^{\prime \prime}\right) F_{0}^{\prime 2}+4\left(F_{0}+F_{0}^{\prime \prime}\right) F_{0}^{\prime \prime} F_{0}-\left(6+\Omega^{2}\right) F_{0}^{3}\right. \\
\left.-F_{0} W_{0}(1, z)^{2}\right] F_{0} \mathrm{~d} z / 2 .
\end{gathered}
$$

In order to obtain (3.41)-(3.42) we have taken into account that $\mathrm{i} U_{0}, \mathrm{i} W_{0}, Q_{0}$ and $F_{0}$ are real (see (3.5), (A9a) and (A.9b)); we have also used (3.32), (3.35) and the equations

$$
\begin{aligned}
& \int_{-\Lambda}^{\Lambda} \int_{0}^{1}\left(U_{0} U_{1}+W_{0} W_{1}\right) r \mathrm{~d} r \mathrm{~d} z=\int_{-\Lambda}^{A}\left[-\alpha_{1}(1-\mathrm{i}) F_{0} Q_{0}(1, z) / \Omega-F_{1} Q_{0}(1, z)\right] \mathrm{d} z, \\
& \int_{-A}^{\Lambda} \int_{0}^{1}\left[\left(U_{0 r}^{2}+U_{0 z}^{2}+W_{0 r}^{2}+W_{0 z}^{2}\right) r+U_{0}^{2} / r\right] \mathrm{d} r \mathrm{~d} z \\
&=4 F_{0}^{\prime}(\Lambda) F_{0}^{\prime \prime}(\Lambda)+\int_{-A}^{\Lambda}\left[\Omega^{2} F_{0}^{2}+2 F_{0}^{\prime \prime} Q_{0}(1, z)\right] \mathrm{d} z
\end{aligned}
$$

which are obtained after some manipulations of the problems giving $\left(U_{0}, W_{0}, Q_{0}, F_{0}\right)$ and $\left(U_{1}, W_{1}, Q_{1}, F_{1}\right)$.

A plot of the constants $\alpha_{2}$ and $\alpha_{3}$ in terms of $A$ is given in figures 4 and 5 for the first four inviscid modes. Notice that for $m=1, \alpha_{3}$ diverges at the capillary instability limit, $A=\pi$, and at $\Lambda \simeq 0.249$ (where $2 \Omega$ is also an inviscid frequency); there are other divergences of $\alpha_{3}$ that either correspond to higher-order modes or to values of $\Lambda$ that are greater than $\pi$.

\subsection{Validity limits of our weakly nonlinear description}

The analysis above breaks down if parametric resonances of second or third order are present, and also if either $\Omega$ is too large or $\Lambda|\pi-\Lambda|$ is too small (we only consider the range $0<A<\pi$ to avoid the capillary instability), as we discuss now. 


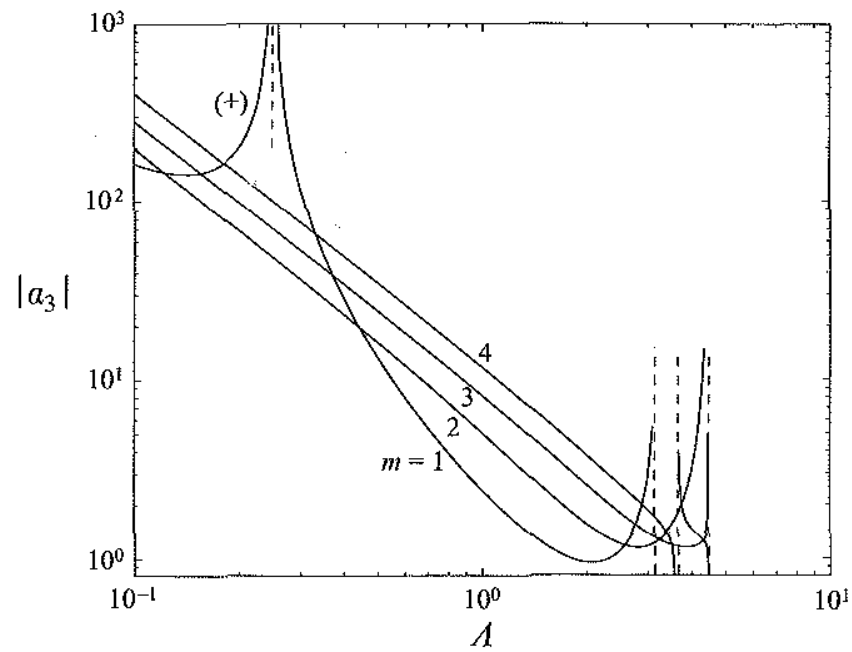

FIGURE 5. The coefficient $\alpha_{3}$ in the cubic term of the amplitude equation ( $3.3 a$ ) for the first four modes. $\alpha_{3}$ is always negative except for the part of the plot corresponding to the first mode that is indicated with the sign $(+)$.

(a) If either $2 \Omega$ or $\Omega / 2$ is also an inviscid eigenfrequency, or more generally, if there are two additional inviscid eigenfrequencies, $\Omega_{1}$ and $\Omega_{2}$, such that $\Omega \pm \Omega_{1} \pm \Omega_{2}=0$, then our analysis is not valid because it ignores the coupled effect of other inviscid modes that may also be excited. In this case, two or three coupled amplitude equations, with quadratic nonlinearities, must be considered that exhibit chaotic behaviour (Mancebo, Nicolás \& Vega 1996). Similarly, if there are three additional inviscid eigenfrequencies, $\Omega_{1}, \Omega_{2}$ and $\Omega_{3}$ such that $\Omega \pm \Omega_{1} \pm \Omega_{2} \pm \Omega_{3}=0$, then we should consider two, three or four coupled amplitude equations, with cubic nonlinearities. Higher-order parametric resonances do not affect the validity of our analysis in the time scales we are considering.

(b) Our analysis also breaks down if there is a second inviscid eigenfrequency such that either $\left|\Omega_{1}-\Omega\right| \sim \delta$ or $\left|\Omega_{1}-\Omega\right| \ll \delta$ (with $\delta=\alpha_{1} C^{1 / 2}+\alpha_{2} C$, see $\S 4$ ) because then the inviscid mode associated with $\Omega_{1}$ is also excited and its coupled effect cannot be ignored. This may in fact occur for high-order inviscid modes as we see now. The frequency of the $m$ th inviscid mode, $\Omega_{m}$, is such that $\Omega_{m}^{2} /(m \pi / 2 A)^{3} \rightarrow 1$ as $m \rightarrow \infty$ (see (A6)); then $\Omega_{m+1}-\Omega_{m} \sim m^{1 / 2}$ as $m \rightarrow \infty$. On the other hand, it may be seen that $\alpha_{1} \sim \Omega^{1 / 2}$ and $\alpha_{2} \sim \Omega^{4 / 3}$ as $\Omega \rightarrow \infty$. Then our analysis is valid only if we are exciting the $m$ th inviscid eigenmode with $m^{3} C^{2} \ll 1$ (for $\Omega_{m+1}-\Omega_{m}$ to be large as compared with $\delta$ ), i.e. with $\Omega_{m} C \ll 1$. Otherwise we must take into account the coupled effect of infinitely many inviscid eigenmodes (with frequencies $\left.\cdots, \Omega_{m-2}, \Omega_{m-1}, \Omega_{m+1}, \Omega_{m+2}, \cdots\right)$, that add up to a pair of counterpropagating, modulated capillary wavetrains that interact nonlinearly and exhibit dispersion and reflection at the disks. Any further discussion on these wavetrains is of course beyond the scope of this paper.

(c) The characteristic size of the Stokes boundary layers is of the order of $(C / \Omega)^{1 / 2}$ (see \$3.2) and must be small compared to $A$ (the size of the liquid bridge) for the analysis above to make sense. That condition fails (in the interval $0<A<\pi$ ) in two limits: as $A \sim C^{2}$ or $A \ll C^{2}$ (because $\Omega \sim A^{-3 / 2}$ as $A \rightarrow 0$ for a fixed inviscid eigenmode, see (A6)); and as $|\pi-\Lambda| \sim C^{2}$ or $|\pi-\Lambda| \ll C^{2}$ if we are exciting the 
first inviscid eigenmode, whose associated eigenfrequency is of the order of $(\pi-\Lambda)^{1 / 2}$ as $A \rightarrow \pi$. We shall not pursue these limits any further because they are somewhat distant from the main object of the paper.

\section{The amplitude equation}

The amplitude equation (3.3b), with $H_{1}, \cdots, H_{6}$ as given by (3.32) and (3.40) may be written as

$$
\varepsilon \delta \frac{\mathrm{d} A}{\mathrm{~d} \tau}=-\varepsilon\left[(1+\mathrm{i}) \alpha_{1} C^{1 / 2}+\alpha_{2} C\right] A+\mathrm{i} \alpha_{3} \varepsilon^{3} A|A|^{2}+\mathrm{i} \mu \alpha_{4}^{+}\left[\beta_{+} \mathrm{e}^{\mathrm{i} \omega_{+} \tau}-(-1)^{m} \beta_{-} \mathrm{e}^{\mathrm{i} \omega_{-} \tau}\right]+\text { HOT, }
$$

where we are using (3.36), $m$ is the order of the mode being excited and

$$
\text { HOT }=O\left(\varepsilon C^{3 / 2}+\varepsilon^{5}+\mu\left(\varepsilon^{2}+C^{1 / 2}\right)\right),
$$

as found after a somewhat careful analysis. The real constants $\alpha_{1}, \alpha_{2}, \alpha_{3}$ and $\alpha_{4}^{+}$are as given by (3.33)-(3.34) and (3.41)-(3.42) and plotted in figures 2-5 for the first four modes. Notice that $\alpha_{1}$ and $\alpha_{2}$ are always positive, while $\alpha_{3}$ and $\alpha_{4}^{+}$may be either positive or negative, depending on the mode being excited and the slenderness.

Notice that the forcing term vanishes if $\omega_{+}=\omega_{-}$(i.e. the forcing frequencies of the disks are equal) and either (i) $m$ is even and $\beta_{+}=\beta_{-}$(i.e. an even mode is excited and the disks are vibrated in phase, with the same amplitude) or (ii) $m$ is odd and $\beta_{+}=-\beta_{-}$(i.e. an odd mode is excited and the disks are vibrated in antiphase, with the same amplitude). In both cases, higher-order terms should be considered in (4.1) to calculate the forcing term, and the complex amplitude evolves on a still larger time scale.

We now define the small parameters $\delta, \varepsilon$ and $\mu$ as

$$
\delta=\alpha_{1} C^{1 / 2}+\alpha_{2} C, \quad \varepsilon=\left(\delta /\left|\alpha_{3}\right|\right)^{1 / 2}, \quad \mu=\varepsilon \delta,
$$

and re-scale the complex amplitude $A$ as

$$
A=\mathrm{i}\left(\beta_{+} B /\left|\beta_{+}\right|\right) \mathrm{e}^{\mathrm{i} \omega_{+} \tau} \quad \text { if } \alpha_{3}>0, \quad A=-\mathrm{i}\left(\beta_{+} \bar{B} /\left|\beta_{+}\right|\right) \mathrm{e}^{\mathrm{i} \omega_{+} \tau} \quad \text { if } \alpha_{3}<0,
$$

to rewrite $(4.1)$ as

$$
\mathrm{d} B / \mathrm{d} \tau=-\left(1+\mathrm{i} \omega_{1}\right) B+\mathrm{i} B|B|^{2}+M\left(1+N \mathrm{e}^{\mathrm{i} \omega_{2} \tau}\right),
$$

where we are ignoring HOT, we are assuming that $\beta_{+} \neq 0$ and

$$
\begin{aligned}
\omega_{1} & =\left(\omega_{+}+\alpha_{1} C^{1 / 2} / \delta\right) \alpha_{3} /\left|\alpha_{3}\right|, \quad \omega_{2}=\left(\omega_{-}-\omega_{+}\right) \alpha_{3} /\left|\alpha_{3}\right|, \quad M=\alpha_{4}^{+}\left|\beta^{+}\right|, \\
N & =(-1)^{m+1} \beta_{-} / \beta_{+} \quad \text { if } \alpha_{3}>0, \quad N=(-1)^{m+1} \bar{\beta}_{-} / \bar{\beta}_{+} \quad \text { if } \alpha_{3}<0 .
\end{aligned}
$$

Notice that the real parameters $M, \omega_{1}$ and $\omega_{2}$ and the complex parameter $N$ may be selected independently by appropriately choosing the forcing frequencies, amplitudes and phases. Two cases must be distinguished.

\subsection{Periodic oscillations: $\beta_{-}=0$ or $\omega_{+}=\omega_{-}$}

If either $N=0$ (i.e. only one disk is vibrated) or $\omega_{2}=0$ (i.e. the vibrating frequencies of the disks are equal) then (4.4) may be written as

$$
\mathrm{d} B / \mathrm{d} \tau=-\left(1+i \omega_{1}\right) B+\mathrm{i} B|B|^{2}+M_{1},
$$

where

$$
M_{1}=M \quad \text { if } N=0, \quad M_{1}=M(1+N) \quad \text { if } \omega_{2}=0 .
$$


We now briefly justify a well-known property of (4.7), namely that its solutions converge to a steady state as $\tau \rightarrow \infty$. To see that we first multiply (4.7) by $\bar{B}$ and add to the resulting equation its complex conjugate to obtain

$$
\mathrm{d}|B|^{2} / \mathrm{d} \tau=-2|B|^{2}+M_{1} B+\bar{M}_{1} \bar{B} .
$$

This equation readily implies that

$$
|B| \leqslant\left|M_{1}\right| \quad \text { as } \tau \rightarrow \infty,
$$

i.e. the orbits of (4.7) are bounded. Then, according to Poincare-Bendixon theorem (Perko 1991), every orbit of (4.7) aproaches either a steady state or a closed curve of the phase plane as $\tau \rightarrow \infty$; but the latter cannot occur, according to Bendixon criterium (Perko 1991) (because if (4.7) is rewritten as a second-order system of real equations, $\mathrm{d} \boldsymbol{x} / \mathrm{d} t=\boldsymbol{f}(\boldsymbol{x})$, then the divergence of the vector field $\boldsymbol{f}$ is $\operatorname{div} \boldsymbol{f}=-2<0$ for all vectors $\boldsymbol{x}$ ).

The steady states of (4.7) are readily calculated by means of the following pair of real equations:

$$
\begin{gathered}
|B|\left[\cos \varphi+\left(|B|^{2}-\omega_{1}\right) \sin \varphi\right]=\left|M_{1}\right|, \\
\left(|B|^{2}-\omega_{1}\right) \cos \varphi-\sin \varphi=0,
\end{gathered}
$$

where $\varphi$ is the phase of $B / M_{1}$. The variable $\varphi$ may be eliminated from (4.10)-(4.11) to obtain the following cubic equation in $|B|^{2}$ :

$$
|B|^{2}\left[1+\left(|B|^{2}-\omega_{1}\right)^{2}\right]=\left|M_{1}\right|^{2} .
$$

The bifurcation diagram of that equation is plotted in figure 6 . The equation possesses exactly three solutions in the multiplicity region, two solutions in the boundary of that region, which is given by

$$
\left|M_{1}\right|^{2}=2\left[\omega_{1}\left(\omega_{1}^{2}+9\right) \pm\left(\omega_{1}^{2}-3\right)^{3 / 2}\right] / 27, \quad \text { with } \omega_{1} \geqslant \sqrt{3},
$$

and a unique solution in the remaining part of the $\left(\omega_{1},\left|M_{1}\right|^{2}\right)$-plane. Also, it is readily seen that when (4.12) has three solutions, $0<|B|_{1}<|B|_{2}<|B|_{3}$, the steady state of (4.7) corresponding to $|B|_{2}$ is unstable, and the other two are asymptotically stable; when only one solution exists, it corresponds to an asymptotically stable steady state of (4.7). If $\left|M_{1}\right|^{2}$ (resp. $\omega_{1}$ ) is kept fixed, the solutions of (4.12) may be plotted in terms of $\omega_{1}$ (resp. $\left|M_{1}\right|^{2}$ ) by means of a curve that is monotonic if $\left|M_{1}\right|^{2}<(4 / 3)^{3 / 2}$ (resp. $\omega_{1}<\sqrt{3}$ ) or S-shaped if $\left|M_{1}\right|^{2}>(4 / 3)^{3 / 2}$ (resp. $\omega_{1}>\sqrt{3}$ ), as shown in figures 7 and 8. For a fixed value of $\left|M_{1}\right|^{2}$, the maximum value of $|B|$ equals $\left|M_{1}\right|$ and it is attained at $\omega_{1}=\left|M_{1}\right|^{2}$.

Finally, notice that the asymptotically stable steady states of (4.7) correspond to orbitally asymptotically stable periodic solutions, of period $2 \pi /\left(\Omega+\delta \omega^{+}\right)$, of (2.1)(2.9) (with the velocity and pressure fields in the bulk and the shape of the free boundary being given by (3.1)).

\subsection{Quasi-periodic oscillations: $\beta_{-} \neq 0$ and $\omega_{+} \neq \omega_{-}$}

Now $N \neq 0$ and $\omega_{2} \neq 0$ in (4.4) (see (4.5) and (4.6)) and this equation must be numerically integrated in order to obtain the large-time behaviour of its solutions. Analytical methods can be used only for some limiting values of the parameters. For example, if $\left|\omega_{2}\right| \ll 1, M$ and $\omega_{1}$ are such that (4.7) exhibits three steady states when $M_{1}=M$, and $|N|$ is sufficiently large for (4.7) to possess a unique steady state when $M_{1}=M(1 \pm|N|)$, then (4.4) exhibits relaxation oscillations; for example, 


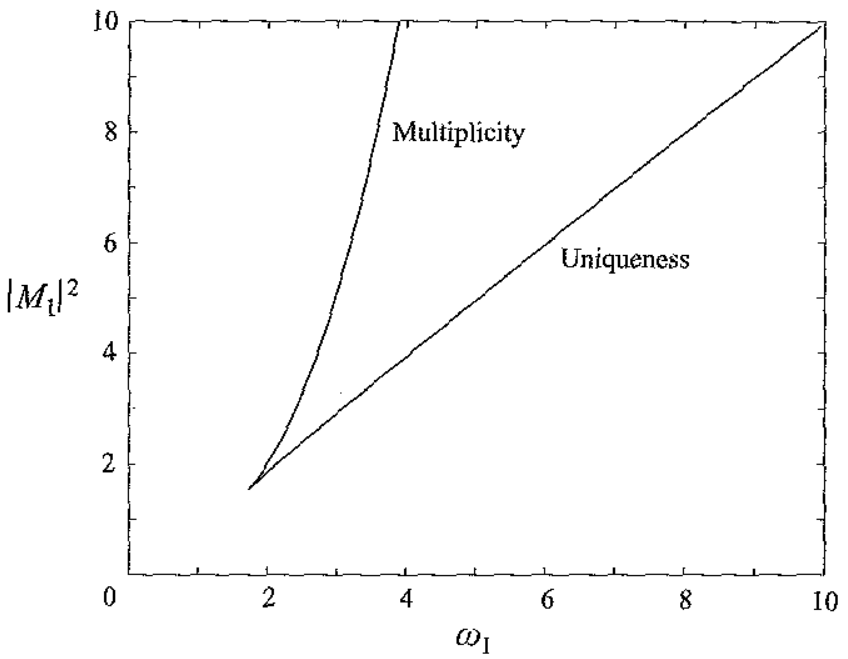

FIGURe 6. Bifurcation diagram of (4.12).

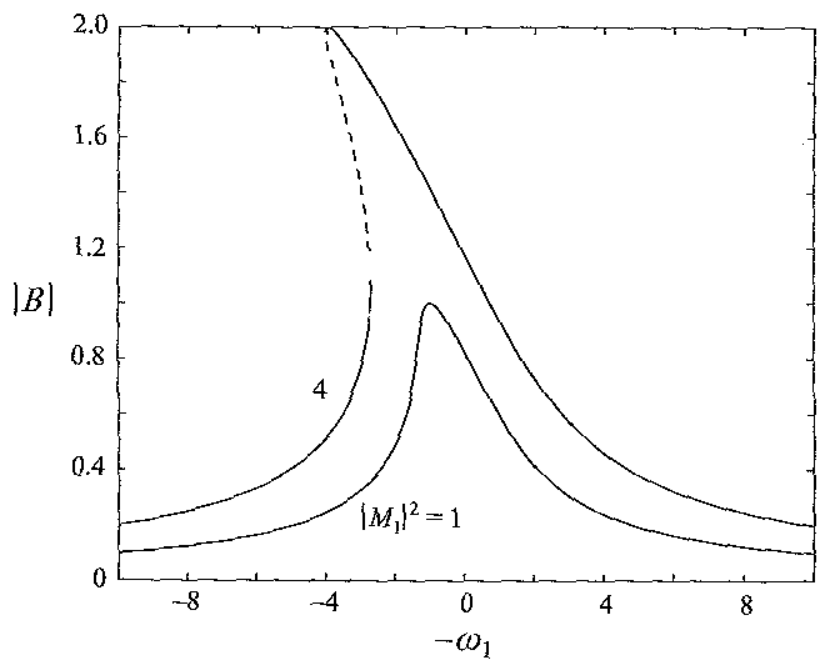

FIGURE 7. Solutions of (4.12) in terms of $\omega_{1}$ for $\left|M_{1}\right|$ fixed. Asymptotically stable and unstable steady states are indicated with solid and dashed lines respectively.

if $\left|\omega_{2}\right| \ll 1, \omega_{1}=3.3, M=2$ and $N=1 / 2$ then that part of the S-shaped curve in figure 8 between $M_{1}=1$ and $M_{1}=3$ is slowly followed (in the time scale $\left.\tau \sim 1 /\left|\omega_{2}\right|\right)$ in the manner indicated by the arrows. These oscillations may be approximately described by means of matched asymptotic expansions (Grasman 1987). For the sake of brevity we do not pursue this (fairly standard) matter any further.

In order to obtain the attractors (as $\tau \rightarrow \infty$ ) of (4.4) for generic values of the parameters we have used a numerical continuation technique (Keller 1987) that allows us to follow continuous branches of periodic solutions when three of the four parameters $\left(\omega_{1}, \omega_{2}, M\right.$ and $\left.N\right)$ are kept fixed and the fourth one is varied. For the sake of brevity we only give a rough description of one of the bifurcation diagrams obtained in this way. 


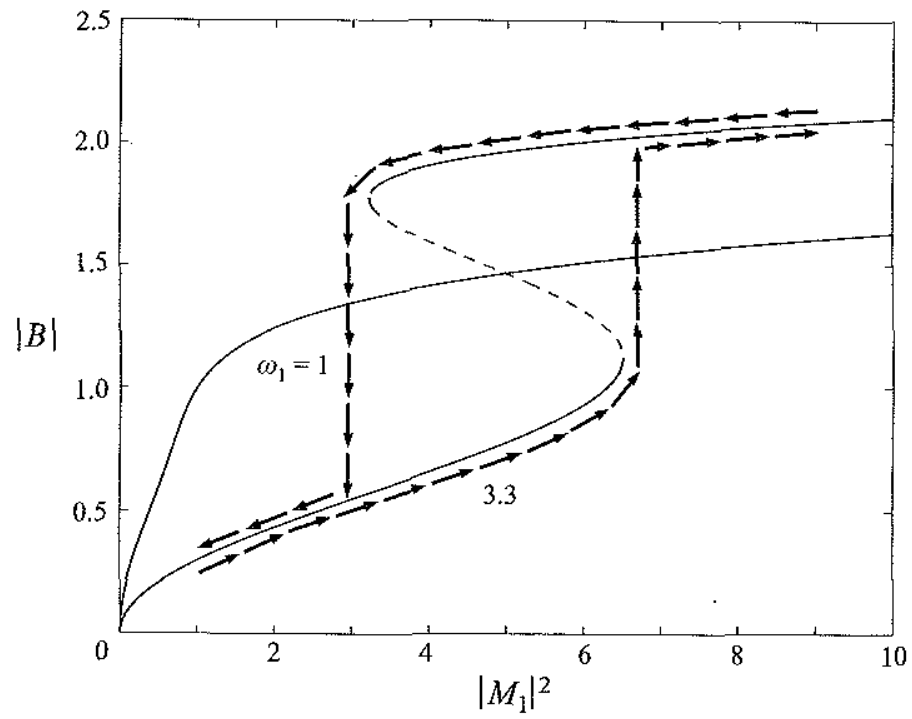

FIGURE 8. Solutions of (4.12) in terms of $\left|M_{1}\right|^{2}$ for $\omega_{1}$ fixed. The arrows indicate the path followed by relaxation oscillations of (4.4) when $\left|\omega_{2}\right| \ll 1, \omega_{1}=3.3, M=2$ and $N=1 / 2$.

That bifurcation diagram corresponds to $\omega_{1}=3.3, M=2$ and $\omega_{2}=-1$, for varying real values of the parameter $N$ (that may be assumed to be real without loss of generality because its phase may be annihilated by means of an appropriate translation in the time variable). If $N=0$ then (4.4) has two exponentially stable steady states, $B=0.217-0.622 \mathrm{i}$ and $B=1.811+0.585 \mathrm{i}$ (and a third unstable steady state). If $0<N<N_{1} \simeq 0.217$ then (4.4) possesses two asymptotically stable periodic solutions of period $2 \pi$; those corresponding to $N=0.21$ are plotted in figure 9. At $N=N_{1}$ that stable periodic solution exhibiting larger values of $|B|$ loses stability through a supercritical period-doubling bifurcation, and a new branch of asymptotically stable $4 \pi$-periodic solutions appears; the asymptotically stable solutions at $N=0.22$ are plotted in figure 10 . At $N=N_{2} \simeq 0.224$ the $4 \pi$-periodic solution exhibits a new supercritical period-doubling bifurcation and if $N_{2}<N<N_{3} \simeq 0.227$ then (4.4) possesses two asymptotically stable periodic solutions, of periods $2 \pi$ and $8 \pi$; those corresponding to $N=0.225$ are plotted in figure 11. At $N=N_{3}$ the bifurcated $8 \pi$-periodic solution disappears through a reversed supercritical period-doubling bifurcation and the branch of $4 \pi$-periodic solutions becomes asymptotically stable again. In the interval $N_{3}<N<N_{4} \simeq 0.271$ several bifurcations of various types take place, which are not considered for the sake of brevity. If $N>N_{4}$ then (4.4) has only one asymptotically periodic solution, of period $2 \pi$. The bifurcation diagram is invariant under the symmetry $N \rightarrow-N$ (because (4.4) is also invariant under the transformation $N \rightarrow-N, \tau \rightarrow \tau+\pi / \omega_{2}$ ). Notice that all periodic solutions of (4.4) considered here generically correspond to quasi-periodic oscillations in the liquid bridge.

\section{Streaming flow in the bulk}

The terms of order $\varepsilon^{2}$ in the expansions (3.1) of the velocity field in the bulk, $u_{2}$ and $w_{2}$, were not completely calculated in $\$ 3$ (see (3.38)) for their slowly varying parts, $u_{20}$ and $w_{20}$, did not affect the amplitude equation. We now calculate $u_{20}$ and $w_{20}$, 


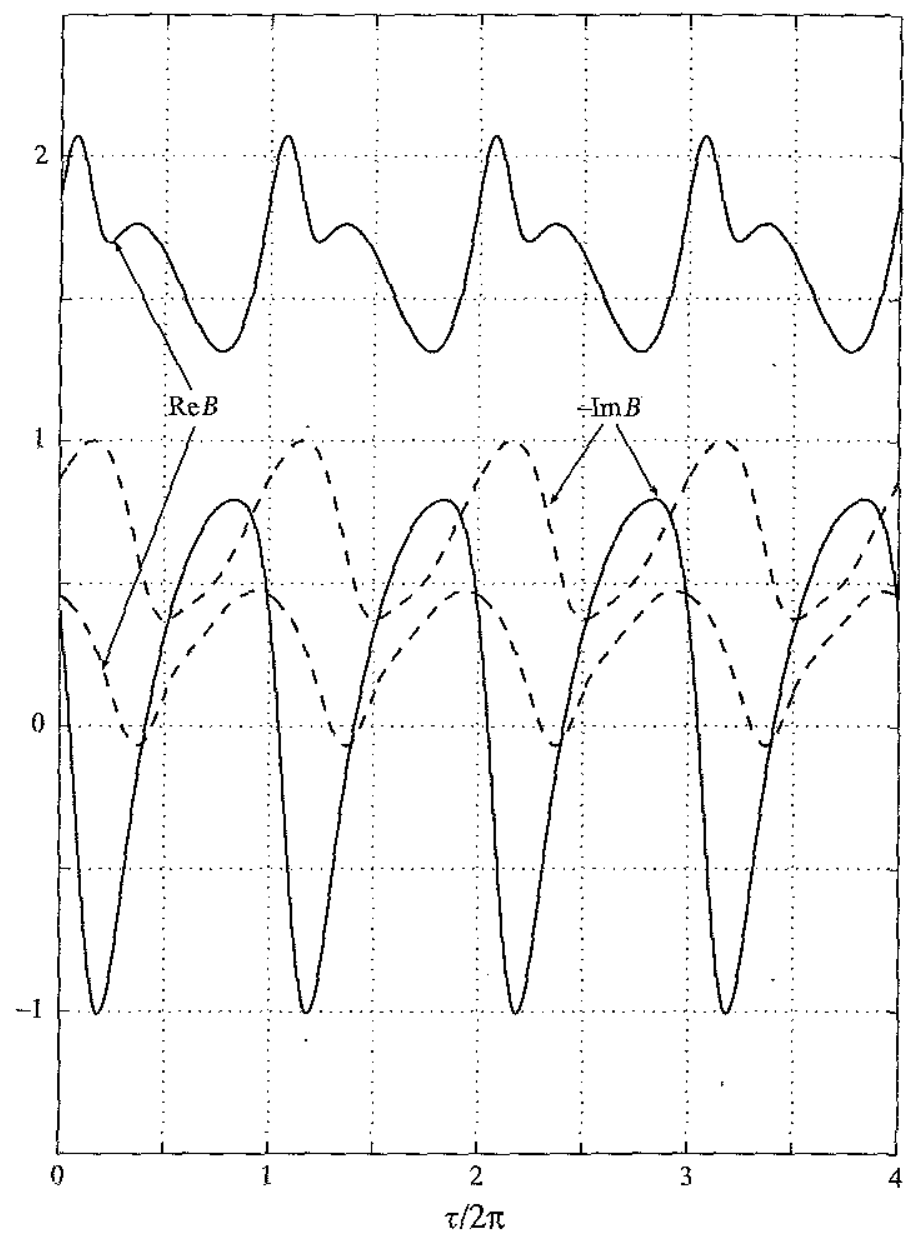

FIGURE 9. Asymptotically stable $2 \pi$-periodic orbits of (4.4) (plotted with solid and dashed lines), for $\omega_{1}=3.3, M=2, \omega_{2}=-1$ and $N=0.21$.

which are independent of the short (capillary) time variable, and may be seen as the leading-order terms of the components of the time-averaged (in the short time scale) velocity field in the bulk; they are given by

$$
\begin{aligned}
& u_{20 r}+r^{-1} u_{20}+w_{20 z}=0, \\
& \left|\alpha_{3}\right| u_{20 \tau}+w_{20}\left(u_{20 z}-w_{20 r}\right)=-q_{70 r}+\gamma\left(u_{20 r r}+u_{20 z z}+r^{-1} u_{20 r}-r^{-2} u_{20}\right), \\
& \left|\alpha_{3}\right| w_{20 \tau}+u_{20}\left(w_{20 r}-u_{20 z}\right)=-q_{70 z}+\gamma\left(w_{20 r r}+w_{20 z z}+r^{-1} w_{20 r}\right), \\
& u_{20}=|B|^{2} g_{1}^{ \pm}(r), \quad w_{20}=0 \quad \text { at } z= \pm A, \\
& u_{20}=w_{20 r}=q_{70 z}=0 \quad \text { at } r=0, \\
& u_{20}=|B|^{2} g_{2}(z), \quad w_{20 r}=|B|^{2} g_{3}(z) \quad \text { at } r=1,
\end{aligned}
$$

where $\tau, \alpha_{3}$ and $B$ are as given in (3.4), (3.42) and (4.2)-(4.3), $q_{70}$ is that part of the term of order $\varepsilon^{4}$ in the expansion (3.1) of the stagnation pressure field in the bulk, $q_{7}$, that is independent of the short time scale $\left(q_{7}=q_{70}+\sum_{k \neq 0} q_{7 k} \mathrm{e}^{\mathrm{i} k \Omega t}\right)$ and the constant $\gamma$, 


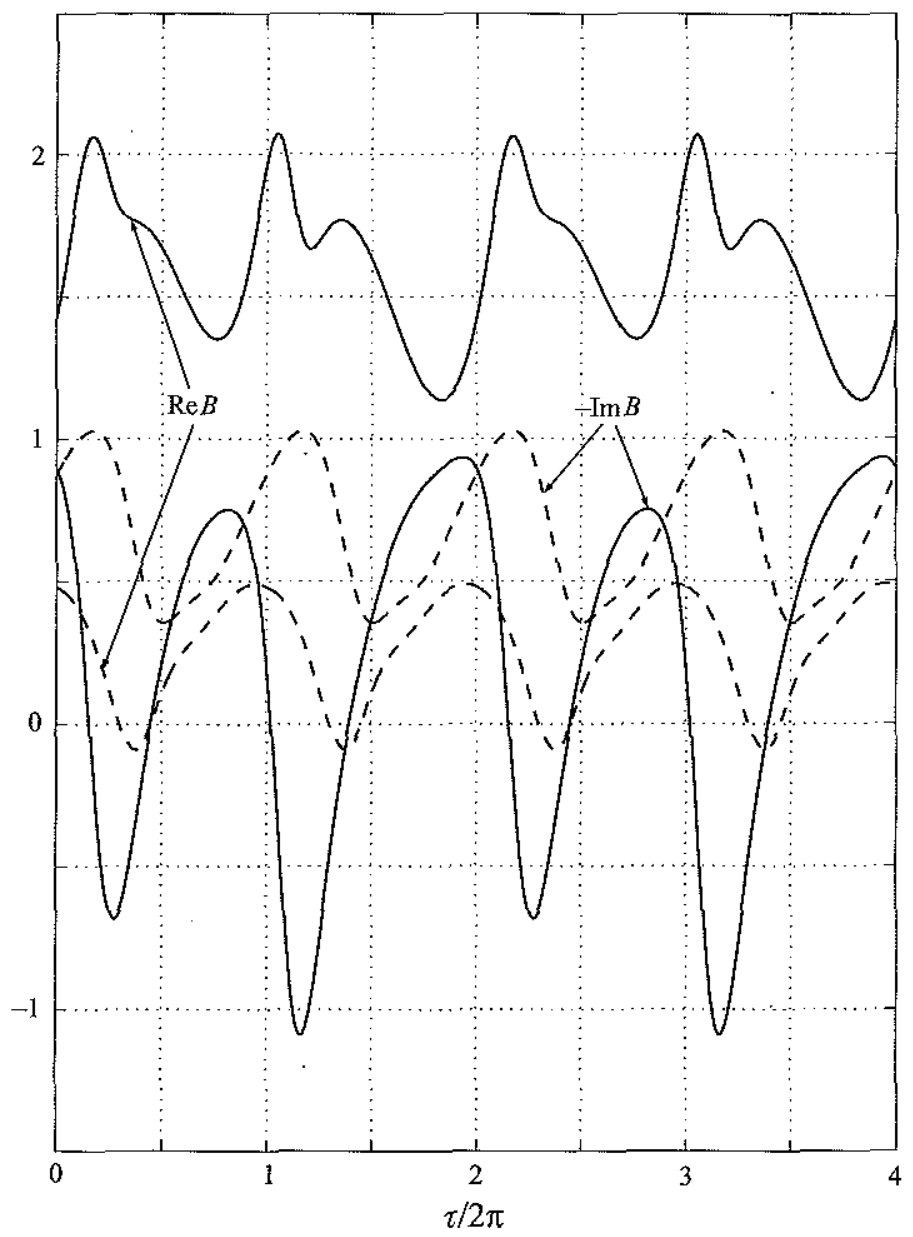

FIGURE 10. Asymptotically stable periodic orbits of (4.4), of periods $2 \pi$ and $4 \pi$ (plotted with dashed and solid lines respectively), for $\omega_{1}=3.3, M=2, \omega_{2}=-1$ and $N=0.22$.

and the functions $g_{1}^{ \pm}, g_{2}$ and $g_{3}$ are given by

$$
\begin{aligned}
& \gamma=\left|\alpha_{3}\right| C /\left(\alpha_{1} C^{1 / 2}+\alpha_{2} C\right), \\
& g_{1}^{ \pm}(r)=-\left[3(1-\mathrm{i}) \bar{U}_{0} U_{0 r}+\text { c.c. }+4 r^{-1}\left|U_{0}\right|^{2}\right]_{z= \pm A} / 2 \Omega, \\
& g_{2}(z)=\left[F_{0}^{\prime} \bar{W}_{0}-F_{0} \bar{U}_{0 r}+\text { c.c. }\right]_{r=1}, \\
& g_{3}(z)=\mathrm{i}\left[3\left(2 \bar{F}_{0}^{\mathrm{iv}}+2 \bar{F}_{0}^{\prime \prime}-\Omega^{2} F_{0}\right) F_{0}^{\prime}+\left(\bar{F}_{0}^{\prime \prime \prime}+\bar{F}_{0}^{\prime}\right) F_{0}^{\prime \prime}\right] / \Omega-\left[\bar{W}_{0 r r} F_{0}\right]_{r=1}+\text { c.c. }
\end{aligned}
$$

Notice that, according to the usual values of $\alpha_{1}, \alpha_{2}$ and $\alpha_{3}$ (see figures 2, 4 and 5), the constant $\gamma$ may be considered as an $O(1)$ quantity for realistically small values of $C$, e.g. $C \gtrsim 10^{-5}$ (even though $\gamma \rightarrow 0$ as $C \rightarrow 0$ ). Equations (5.1)-(5.3) and (5.5) are obtained upon substitution of (3.1) into (2.1)-(2.3) and (2.5), setting to zero that part of the terms of order $\varepsilon^{2}$ in the resulting equations that is independent of the short time variable, and taking into account the definitions (4.2). The boundary conditions (5.4) and (5.6) are readily obtained when taking into account (3.21), (3.22), (3.27) and (3.29). 


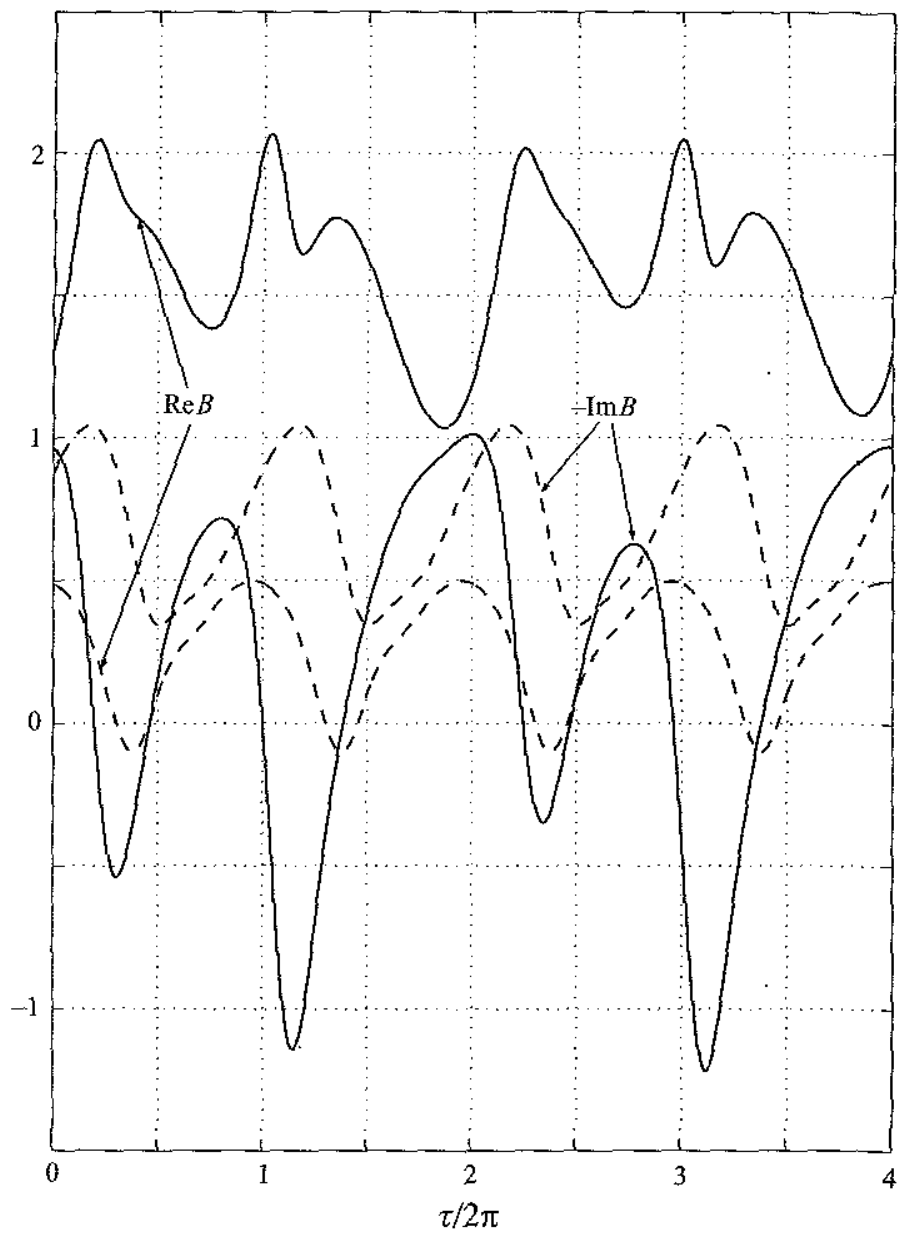

FIGURE 11. As in figure 10 but now the periods are $2 \pi$ and $8 \pi$, and $N=0.225$.

In order to obtain (5.2) and (5.3) we have taken into account that

$$
\begin{aligned}
& \mathrm{i} \Omega^{-1}|A|^{2} U_{0} \bar{W}_{0}\left[r^{-1}\left(w_{20 r}-u_{20 z}\right)-\left(w_{20 r}-u_{20 z}\right)_{r}\right]+\text { c.c. } \equiv 0, \\
& -\left[\mathrm{i} \Omega^{-1}|A|^{2} U_{0} \bar{W}_{0}\left(w_{20 r}-u_{20 z}\right)_{z}+\text { c.c. }\right] \equiv 0,
\end{aligned}
$$

as readily seen when taking into account that, according to (3.5), (A7)-(A9a) and $(\mathrm{A} 9 \mathrm{~b}), U_{0}$ and $W_{0}$ are purely imaginary. If the left-hand sides of these two equations were non-zero (as happens to be the case for non-resonant oscillations, see Nicolás et al., 1996a) then they should be added to the left-hand sides of (5.2) and (5.3) respectively; these two additional terms are obtained as the short-time averages of $\left(A W_{0} \mathrm{e}^{\mathrm{i} \Omega t}+\right.$ c.c. $)\left(u_{5_{z}}-w_{5 r}\right)$ and $\left(A U_{0} \mathrm{e}^{\mathrm{i} \Omega t}+\right.$ c.c. $)\left(w_{5 z}-u_{5 r}\right)$ respectively, where $u_{5}=\mathrm{i} \Omega^{-1} A W_{0} \mathrm{e}^{\mathrm{i} \Omega t}\left(u_{20 z}-w_{20 r}\right)+$ c.c. $+q_{5 r}+$ NRT and $w_{5}=\mathrm{i} \Omega^{-1} A U_{0} \mathrm{e}^{\mathrm{i} \Omega t}\left(u_{20 r}-w_{20 z}\right)+$ c.c. $+q_{s z}+$ NRT are the components of the velocity field at order $\varepsilon^{3}$ (and NRT stands for non-resonant terms, as in the Appendix).

Since $U_{0}$ is either symmetric or antisymetric in the $z$-variable, $F_{0}$ is real and $U_{0}$ and $W_{0}$ are purely imaginary (see (3.5), (A9a) and (A9b)), we have

$$
g_{1}^{+}(r) \equiv g_{1}^{-}(r), \quad g_{2}(z) \equiv g_{3}(z) \equiv 0 .
$$




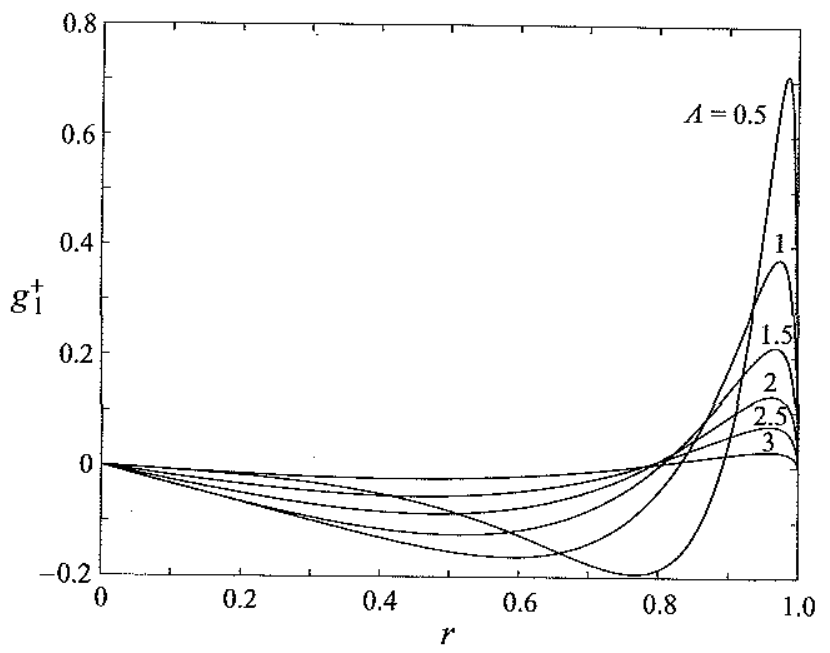

Frgure 12. The function $g_{1}^{+}=g_{1}^{-}$associated with the first natural mode, giving the forcing radial velocity in the boundary condition (5.4), for several values of the slenderness.

In adition, the net tangential radial flux at the disks is seen to satisfy

$$
|B|^{2} \int_{0}^{1} r g_{1}^{ \pm}(r) \mathrm{d} r=-|B|^{2} \int_{0}^{1}\left|U_{0}(r, \Lambda)\right|^{2} \mathrm{~d} r / 2 \Omega<0 .
$$

A plot of the forcing term $g_{1}^{+}=g_{1}^{-}$associated with the first natural mode is given in figure 12 for several values of the slenderness.

The model (5.1)-(5.6) provides the (Eulerian) short-time-averaged velocity that, for kinematical reasons, needs not coincide with the drift (or mass-transport) velocity, that is, the (Lagrangian mean) velocity associated with the short-time-averaged trajectories of material elements; notice that the drift velocity must be used for comparison with experiments. But, since our oscillating field is standing (that is, its phase is independent of position) in first approximation, both velocities do coincide (Batchelor 1967) and (5.1)-(5.6) also provides the drift velocity field.

Now, if either (i) only one disk is vibrated or (ii) both disks are vibrated with the same frequency then, as seen in $\S 4.1,|B|$ evolves to a steady state as $\tau \rightarrow \infty$. If the initial transient is ignored, then $|B|^{2}$ may be considered as a constant in (5.4) (that may be calculated, as a steady state of (4.7), in terms of the non-dimensional frequency detuning $\omega_{1}$ and forcing amplitude $\left|M_{1}\right|$; recall that (4.7) may exhibit up to three steady states). If, in addition, $|B|^{2}$ is not too large (to avoid flow instabilities) then the solution of (5.1)-(5.6) evolves to a steady state as $\tau \rightarrow \infty$. If the eigenmode being excited and the slenderness are kept fixed, then the forcing function $g_{1}^{ \pm}$remains constant and that steady state depends only on the parameter

$$
k=|B|^{2} / \gamma,
$$

as readily seen when dividing $u_{20}$ and $w_{20}$ by $\gamma$, and $q_{70}$ by $\gamma^{2}$ in (5.1)-(5.6).

The steady state has been numerically calculated for five values of the constant $k$ (which may be seen as an effective Reynolds number associated with the steady streaming flow), $k=2,20,200,1000$ and 2000 , and for $A=1$; in all cases we have considered the forcing term $g_{1}^{ \pm}$associated with the first natural mode (see figure 12). 


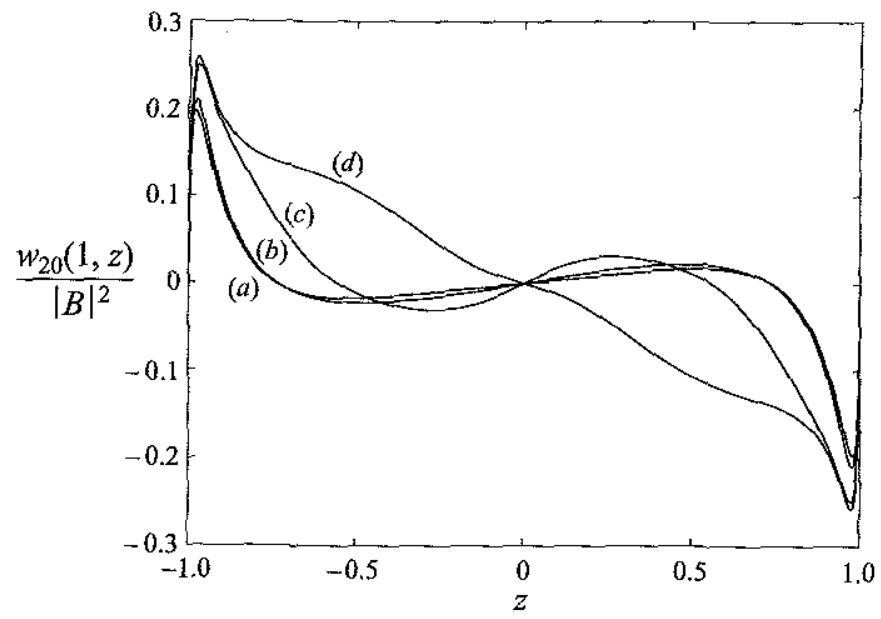

FIGURE 13. Axial velocity at the interface for the steady streaming due to the first mode at $A=1$. (a) $(---) k=2,(\longrightarrow) k=20$ (indistinguishable), (b) $k=200,(c) k=1000,(d) k=2000$.

The resulting axial velocity at the interface, $w_{20}(1, z) /|B|^{2}$, and the streamlines are plotted in figures 13 and 14. Several remarks concerning these results are in order.

(a) Both the axial velocity at the interface and the streamlines in figures 13 and 14 are quite representative of other numerical results obtained for higher-order natural modes and for other values of the slenderness.

(b) The flow pattern is always symmetric on the plane $z=0$; thus only one half of the liquid bridge is plotted in figure 14, even though numerical calculations were made in the whole domain, $0<r<1,-1<z<1$, to ensure that no symmetry breaking appears in the range of $k$ considered.

(c) The quantity $w_{20} /|B|^{2}=w_{20} / \gamma k$ and the streamlines are almost equal for $k=2$ and 20 (see figures 13 and $14 a$ ). This means that $u_{20} / \gamma$ and $q_{20} / \gamma^{2}$ depend linearly on $k$ if $0 \leqslant k \leqslant 20$ and thus satisfy the (Stokes) linear problem resulting from neglecting convective terms. This was to be expected because, in two-dimensional incompressible flows in finite containers resulting from steady forcing at the boundary, the zero-Reynolds-number limit frequently applies up to fairly large values of the Reynolds number.

(d) For $k=2,20$ and 200 the flow pattern exhibits two small toroidal eddies near the corners, $r=1, z= \pm 1$, that push the liquid away from the disks along the interface, and two larger counter-rotating (toroidal) eddies in the bulk (that push the liquid towards the disks along the interface). Again, this was to be expected; the small eddies are due to the fact that the forcing radial velocity at the disks is positive in a small region near the corners (see figure 12), and the large eddies are due to the fact that the net forcing radial flux is negative (see (5.11)). These flow patterns are in rough qualitative agreement with the experimental observations by Mollot et al. (1993, figure 12).

(e) For larger values of $k$ the small eddies near the corners enlarge (see figure $14 c$ ), due to convective effects, to cover the whole interface (as in figure 14d). These two flow patterns were not observed by Mollot et al. (1993). 
(a)

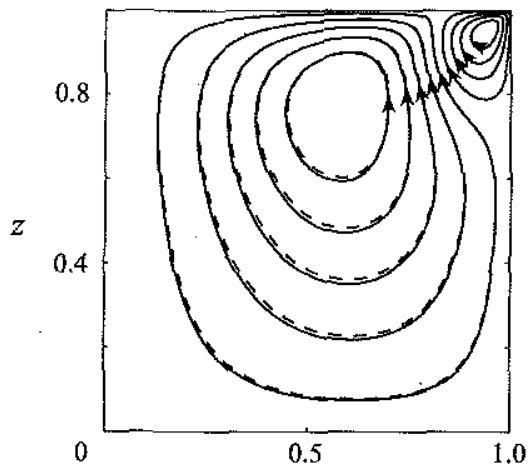

(c)

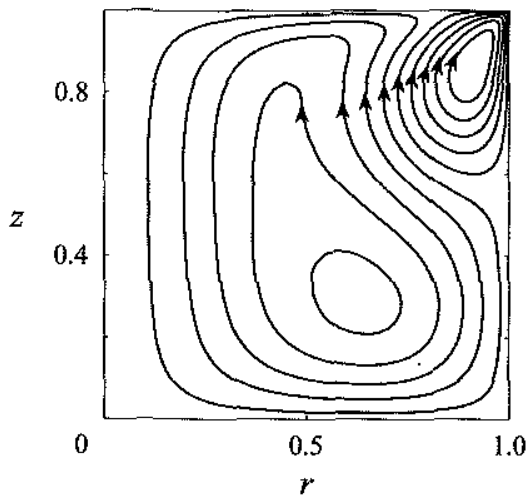

(b)

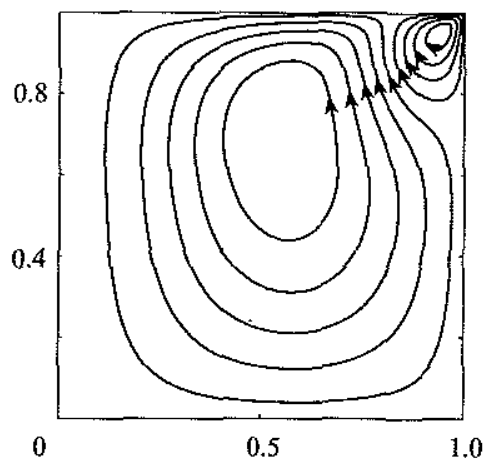

$(d)$

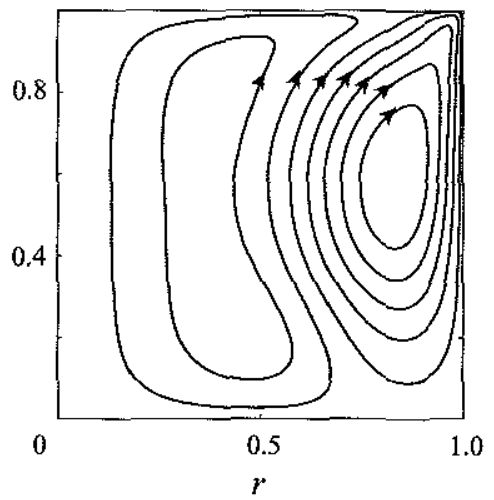

FIGURE 14. Streamlines for the five cases in figure 13. Flow patterns are always symmetric and only one half of the capillary bridge is plotted.

\subsection{The combined effect of vibration and thermocapillary stress}

Let us now consider the combined effect of the steady forcing terms due to vibration and the thermocapillary stress in non-isothermal conditions. We shall consider a distinguished limit when $(a)$ the temperature is independent of the fast (capillary) time $t$ in first approximation, and $(b)$ thermocapillary stress produces a velocity field in the bulk that is of the same order as that associated with the streaming flow considered above (that is, of the order of $\varepsilon^{2}$ ). As a consequence $(c)$ the oscillating flow considered in $\S \S 3$ and 4 (that is governed by the amplitude equation (4.1)) will be independent of thermocapillary effects (as it was independent of the streaming flow); and $(d)$ convection and thermal diffusion will be of the same order in the energy equation if the Prandtl number is of order unity and the basic thermal state will depend on the slowly varying flow even to the leading order. In this limit we shall derive an asymptotic model that gives both the slowly varying temperature field and the slowly-varying flow (resulting from both thermocapillary effects and streaming effects due to vibration). The steady states of this model will be analysed in order to inquire into whether vibration can counterbalance thermocapillary effects. For the sake of completeness we shall maintain below the dependence on the slow time variable $\tau$; then our asymptotic model may be used also to analyse the stability of the steady flows considered below and some more complex dynamics that will not be 
considered. But the derivation below could be more easily followed if the dependence on the slow time variable is ignored. For the sake of brevity, the derivation below is not given in full. See Nicolás et al. (1996a) for a more detailed derivation of a related asymptotic model that exhibits the same features as that below and applies to the non-resonant case.

In order to model thermocapillary effects, the tangential stress balance at the interface (2.7) must be replaced by

$$
\left(w_{r}+u_{z}\right)\left(1-f_{z}^{2}\right)+2\left(u_{r}-w_{z}\right) f_{z}=-(C \operatorname{Re})\left(1+f_{z}^{2}\right)^{1 / 2}\left(\theta_{z}+f_{z} \theta_{r}\right) \quad \text { at } \quad r=f,
$$

where $R e$ is the effective (or thermocapillary) Reynolds number, defined as $R e=$ $\sigma_{T}(\Delta T) R / \rho v^{2}$ (with $\Delta T$ a typical temperature increment in the liquid bridge, $\sigma_{T}=$ $\partial \sigma / \partial T$ the thermal gradient of surface tension, $\rho$ the density, $v$ the kinematic viscosity and $R$ the radius of the disks, as defined in $\$ 2)$, and $\theta$ is a non-dimensional temperature $\left(\theta=\left(T-T_{0}\right) /\left(T_{1}-T_{0}\right)\right.$, where $T_{0}$ is the temperature of the disks and $T_{1}$ is a characteristic temperature in the liquid bridge), that satisfies the energy conservation equation

$$
\theta_{t}+u \theta_{r}+w \theta_{z}=(C / P)\left(\theta_{r r}+r^{-1} \theta_{r}+\theta_{z z}\right)
$$

and boundary conditions

$$
\theta=0 \quad \text { at } z= \pm A, \quad \theta_{r}-f_{z} \theta_{z}=\left(1+f_{z}^{2}\right)^{1 / 2} g(z) \quad \text { at } r=f .
$$

Here $P=\rho \nu c_{p} / k_{0}\left(c_{p}\right.$ is specific heat, $k_{0}$ is thermal conductivity) is the Prandtl number and $g=g(z)$ is the non-dimensional normal heat flux through the interface, that is assumed to be given and symmetric, i.e. $g(-z) \equiv g(z)$. As is usual in the literature, we are assuming all properties of the liquid to be independent of temperature except for surface tension, which depends linearly on temperature in first approximation. That dependence is taken into account only in the tangential stress balance boundary condition at the interface (where that dependence appears at leading order, in the right-hand side of (5.13), and not as a correction to something larger). The thermal boundary conditions (5.15) are the simplest ones giving symmetric thermocapillary flows, which are of interest in materials processing as indicated in $§ 1$.

We shall consider the distinguished limit

$$
\operatorname{Re} \sim P \sim 1,
$$

but our asymptotic model below may be seen to apply to larger values of the Reynolds number, namely whenever

$$
R e \ll C^{-1} \text {. }
$$

Notice that if this condition holds then the velocity and pressure fields associated with the thermocapillary stress are such that $u, w$ and $q$ are small (even though the thermocapillary Reynolds number may be large) and the thermocapillary flow can be considered as a perturbation of the quiescent state when analysing the oscillatory flow (with the non-dimensionalization based on the capillary characteristic time we are using in this paper); thus the analysis in $\S 3$ remains valid (except for the boundary condition (5.20) below).

Now, the temperature in the bulk is expanded as

$$
\theta=\theta_{0}(r, z, \tau)+\varepsilon\left[A \Theta_{1} \mathrm{e}^{\mathrm{i} \Omega t}+\text { c.c. }\right]+\text { HOT }
$$

where the basic thermal state $\theta_{0}$ is to be calculated and varies only in the slow time scale $\tau \sim 1$ (where $\tau$ is given by (3.4) and (4.2)), that is the time scale associated with both the damping of the oscillating flow (see (4.4)) and the streaming flow (see 
(5.2)-(5.3)). Notice that (even though we are interested only in the leading-order term $\theta_{0}$ ) we also need to consider a first correction of order $\varepsilon$, that oscillates in the fast (capillary) time scale, $t \sim 1$. If (3.1) and (5.17) are inserted into (5.14) and the coefficient of $\varepsilon$ is set to zero, then $\Theta_{1}$ is seen to be as given by

$$
\Theta_{1}=\mathrm{i} A\left(U_{0} \theta_{0 r}+W_{0} \theta_{0 z}\right) / \Omega \text {. }
$$

Notice that temperature oscillations in the bulk are driven only by the oscillating velocity field through convective effects; heat conduction comes into play to calculate temperature oscillation only in the oscillatory boundary layers (i.e. in regions $(a)$ and $(b)$, see figure 1). Similarly if the non-oscillatory part (i.e. that part that is independent of the fast time variable $t$ ) of the coefficient of $\varepsilon^{2}$ is set to zero, and the definition (4.2) of $\varepsilon$ is used, then we obtain

$$
\theta_{0 z}+u_{20} \theta_{0 r}+w_{20} \theta_{0 z}=(\gamma / P)\left(\theta_{0 r r}+r^{-1} \theta_{0 r}+\theta_{0 z z}\right),
$$

where $\gamma$ is as defined in (5.7) (recall that $\gamma$ may be considered as a $O(1)$ quantity in practice); thus, in the limit (5.16) $\gamma / P$ may be considered as an $O(1)$ quantity and, according to (5.19), the time scale $\tau \sim 1$ coincides with the thermal time scale. In order to obtain (5.19), we have taken into account that $\bar{A}\left(\bar{U}_{0} \Theta_{1 r}+\bar{W}_{0} \Theta_{1 z}\right)+$ c.c. $=0$, as readily seen when using (5.18) and the fact that $U_{0}$ and $W_{0}$ are purely imaginary (see (3.4), (A9a) and (A9b)) and $\theta_{0}$ is real. If this additional term were not zero (as happens when the oscillations are non-resonant, see Nicolás et al., 1996a) then it should be added to the left-hand side of (5.19); notice that this term is obtained as the short-time average of $\left(A U_{0} \mathrm{e}^{\mathrm{i} \Omega t}+\right.$ c.c. $)\left(\Theta_{1 r} \mathrm{e}^{\mathrm{i} \Omega t}+\right.$ c.c. $)+\left(A W_{0} e^{\mathrm{i} \Omega t}+\right.$ c.c. $)\left(\Theta_{1 z} \mathrm{e}^{\mathrm{i} \Omega t}+\right.$ c.c. $)$. Finally, when using the new boundary conditions (5.13) and (5.15) in the analysis of the Stokes and the interface boundary layers (see $\$ \S 3.2$ and 3.3), and applying the appropriate matching conditions with the solution in the bulk, then we obtain the new boundary conditions

$$
\begin{gathered}
u_{20}=0, \quad w_{20 r}=-\gamma \operatorname{Re} \theta_{0 z} \quad \text { at } r=1, \\
\theta_{0}=0 \quad \text { at } z= \pm \Lambda, \quad \theta_{0 r}=g(z) \quad \text { at } r=1,
\end{gathered}
$$

where we have used the definition of $\varepsilon$ (in (4.2)) and (5.10).

Now, the model posed by (4.7), (5.1)-(5.5.) and (5.19)-(5.21), with $\omega_{1}, M_{1}, \gamma$ and $g_{1}^{ \pm}$ as given by (4.5), (4.8) and (5.7), gives the combined effect of the slowly varying forcing terms due to vibration and thermocapillary stress. For the numerical applications below we assume the non-dimensional heat flux through the boundary to be given by a Gaussian, i.e.

$$
g(z)=\exp \left(-a z^{2} / 2\right)
$$

as usually done in the literature to model the heat flux through the interface when considering applications to the float zone technique.

As above, we shall consider only steady flow patterns. Then we may consider $B$ as a parameter (that may be calculated as a steady state of (4.7)). Also, the steady states depend only on the parameters $k$ (see (5.12)) $P$ and $R e$, and on the forcing functions $g_{1}^{ \pm}$(see (5.7) and figure 12) and $g$ (see (5.22)).

In the absence of vibration (namely if $k=0$ ) the temperature attains its maximum at the centre of the liquid bridge $z=0$ (where the heat flux is largest, see (5.22)) and the thermocapillary tangential stress (see (5.20)) pushes the liquid along the interface from the centre of the capillary bridge to the disks. The resulting flow pattern is qualitatively similar to that in figure $14(a, b)$, except for the two small eddies near the corners that are now absent. Thus, the thermocapillary flow is reinforced by vibrations 

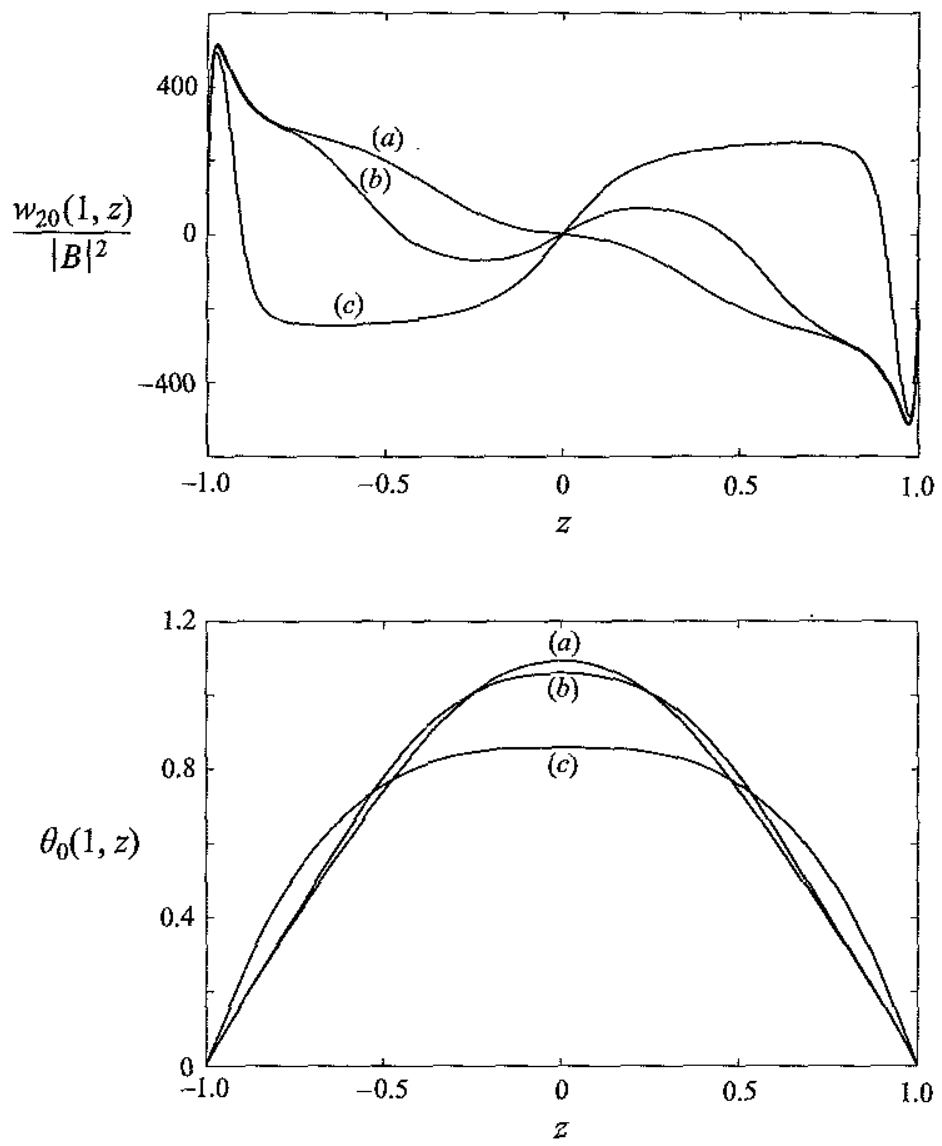

FIGURE 15. Axial velocity and temperature at the interface for the steady flow in the bulk at $A=1$ due to vibration of the first mode with $k=2000$ and thermocapillary effects with $P=0.02:(a)$ $\operatorname{Re}=100,(b) \operatorname{Re}=500$ and (c) $\operatorname{Re}=2000$.

(except near the corners, but this is a localized effect) if $k \leqslant 200$. That prediction is confirmed by some numerical calculations that are not presented for the sake of brevity.

For larger values of $k$ (e.g. $k=2000$ ), counterbalancing is (a priori) possible because the streaming flow due to vibration and the purely thermocapillary flow exhibit essentially opposite structures near the interface. In order to ellucidate whether effective counterbalancing is feasible, the problem (5.1)-(5.5), (5.19)-(5.22) has been numerically integrated for $A=1, P=0.02, k=2000, a=2$ and three values of the thermocapillary Reynolds number, $R e=100,500$ and 2000 . The axial velocity and the temperature at the interface and the streamlines are plotted in figures 15 and 16. Two remarks concerning these results are in order:

(a) As in figures 13 and 14, these flow patterns are representative of counterbalancing results for other (non-limiting) values of the parameters. Also, the flow pattern is always symmetric in figure 16 , where only one half of the liquid bridge has been plotted.

(b) For small thermocapillary effects the flow pattern remains qualitatively unchanged (compare figures $14 d$ and $16 a$ ). As the capillary Reynolds number increases the fluid is pushed more and more towards the disks along the interface by thermo- 
(a)

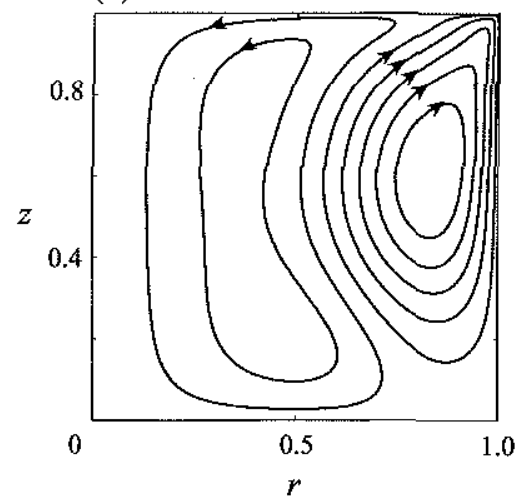

(b)

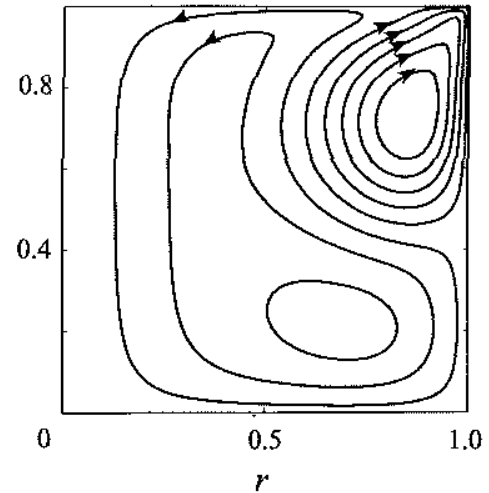

(c)

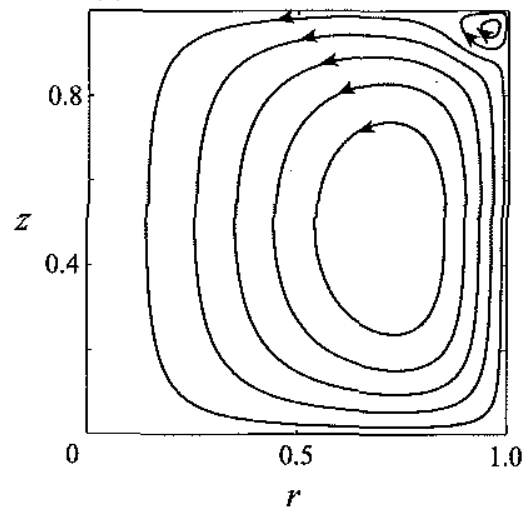

FIGURE 16. Streamlines for the three cases in figure 15. Flow patterns are always symmetric and only one half of the capillary bridge is plotted.

capillary stress until the eddies near the corner become quite localized (see figure $16 \mathrm{c}$ ). Nevertheless the maximum value of the velocity at the interface remains unaffected (see figure 15). This is due to the fact that the boundary condition at the disks, (5.4), is independent of thermocapillary effects, and illustrates the main difficulty that makes these streaming flows inconvenient to counterbalance thermocapillary convection in the whole liquid bridge. Namely, the price for any possible counterbalancing effect in the bulk is the introduction of large velocities near the corners.

Therefore nearly inviscid, almost-resonant, low-frequency vibration is not effective to counterbalance thermocapillary flows in liquid bridges. In order to get effective counterbalancing, the forcing tangential stress at the interface coming from vibration, $w_{20 r}(1, z)=|B|^{2} g_{3}(z)$ (see (5.6)) plays an essential role; it vanishes here (see (5.10)) because the oscillating flow is standing in first approximation and the phase of the complex eigenfunctions $F_{0}$ and $W_{0}$ is independent of the axial coordinate, and this is an exclusive property of almost-resonant, low-frequency vibration. Nonresonant oscillations produce vibrating velocity fields whose phase varies with the axial coordinate when both disks are vibrated with appropriately different phases; these progressive oscillations are quite effective in counterbalancing thermocapillary convection in model-half-zone liquid bridges (whose disks are held at two different temperatures, with the interface thermally insulated), as shown in Nicolás et al. 


$\begin{array}{lrrlcl} & \text { Water } & \text { Mercury } & \text { Water } & \text { Mercury } \\ 10^{3} \times C & 3.3 & 0.6 & 10^{4} \times \alpha(\mathrm{cm}) & 9.3 & 2.0 \\ 10^{3} \times \delta & 28.0 & 6.4 & 10^{3} \times t_{c}(\mathrm{~s}) & 3.7 & 5.3 \\ 10^{2} \times \epsilon & 11.0 & 5.1 & 10^{-2} \times \widetilde{\Omega}(\mathrm{Hz}) & 1.23 & 0.86 \\ 10^{4} \times \mu & 31.0 & 3.3 & 10^{2} \times \Delta f_{m}^{0}(\mathrm{~cm}) & 2.1 & 1.9 \\ 10^{-1} \times k & 5.9 & 28.0 & 10 \times v_{s}\left(\mathrm{~cm} \mathrm{~s}^{-1}\right) & 10.6 & 6.3 \\ |A| & 4.0 & 8.0 & 10 \times v_{l}\left(\mathrm{~cm} \mathrm{~s}^{-1}\right) & 1.6 & 0.9 \\ |\beta+| & 1.5 & 3.0 & & & \end{array}$

TABLE 1. Vibrating parameters and steady streaming results for $A=1, R=1 \mathrm{~mm}$, if the first eigenmode is excited by vibration of one disk.

$(1996 a)$. Also, if the vibrating frequency is large enough to excite a large number of capillary eigenmodes (i.e. $\Omega \sim C^{-1} \gg 1$ ) then these modes add up to a pair of progressive counterpropagating wavetrains along the interface, which have been analysed by Nicolás et al. (1996b), who have shown that the associated streaming flow is quite effective in counterbalancing symmetric and non-symmetric thermocapillary flows. In both cases the steady tangential stress at the boundary is non-zero and may be selected to have the right shape (by appropriately choosing the vibrating parameters) to counterbalance thermocapillary stress.

\section{An application in laboratory dimensional parameters}

For illustration, let us consider two capillary bridges, of water and mercury ( $\rho=1$ and $13.63 \mathrm{gm} \mathrm{cm}^{-3}, \sigma=72$ and 484 dynes $\mathrm{cm}^{-1}$ and $v=0.89 \times 10^{-2}$ and $0.113 \times$ $10^{-2} \mathrm{~cm}^{2} \mathrm{~s}^{-1}$ respectively, at $25^{\circ} \mathrm{C}$ ). If the radius of the disks is $R=1 \mathrm{~mm}$ (a typical value for millimetric liquid bridges) then the small parameter $C=\left(\rho v^{2} / \sigma R\right)^{1 / 2}$ is as given in table 1. If, in addition, the slenderness is $A=1$ and the first eigenmode is excited then $\Omega \simeq 3.3$ (obtained from the first equation of (A6), in the Appendix), the coefficients in the amplitude equations are $\alpha_{1} \simeq 0.09, \alpha_{2} \simeq 7, \alpha_{3} \simeq-2.5$ and $\alpha_{4}^{+} / \Omega^{2} \simeq 0.25$ (see figures 2-5)), and the small parameters $\delta, \varepsilon$ and $\mu$ are as given in table 1 (and obtained from (4.2)). Now, for our analysis to be valid, $\varepsilon|A| \ll 1$. Then we take $\omega_{1}=\left|M_{1}\right|^{2}=16$ and 64 respectively in equation (4.12), whose upper solution (there is also a lower, stable solution at these values of $\omega_{1}$ and $\left|M_{1}\right|$ ) is $|B|^{2}=\left|M_{1}\right|^{2}=16$ and 64 respectively. If only the upper disk is vibrated then $M=\left|M_{1}\right|$ and $|A|=|B|$ (see (4.3) and (4.8)), and $\beta^{+}$and $k$ are readily calculated from (4.5), (5.7) and (5.12), to be as given in table 1 . Now, the dimensional vibrating amplitude, $\alpha=2 \mu\left|\beta_{+}\right| R$ (see (2.11)), the characteristic time $t_{c}=\left(\rho R^{3} / \sigma\right)^{1 / 2}$ and the dimensional vibrating frequency, $\widetilde{\Omega}=\left(\Omega+\delta \omega^{+}\right) / 2 \pi t_{c}$ (with $\omega^{+}$as given by $(4.5)$ ), are readily calculated. Similarly, since the maximum of the eigenfunction $F_{0}$ (given by (A7) in the Appendix) equals 0.235 , the maximum deflection of the interface in one oscillation is $\Delta f_{m}^{0}=2 \varepsilon|A|\left|F_{0}\right|_{\max } R$. Finally, with these small values of the parameter $k$ (59 and 280) the streamlines of the associated streaming flow are qualitatively similar to those in figure $14(a, b)$, and the velocities in the small and large eddies in dimensional terms are $v_{s}=\varepsilon^{2}|B|^{2} w_{20}^{1} R / t_{c}$ and $v_{l}=\varepsilon^{2}|B|^{2} w_{20}^{2} R / t_{c}$ respectively, with $w_{20}^{1} \simeq 0.2$ and $w_{20}^{2} \simeq 0.03$ (see figure 13).

Notice that we are obtaining fairly large streaming velocities with very small oscillatory deflection of the interface. Our results suggest that only the first two patterns in figure 14 are obtained in millimetric liquid bridges if we maintain the 
condition of small oscillatory deflection of the interface (that requires $\varepsilon|A|$ to be appropriately small), excite the first mode and take $A \sim 1$ (the physical properties of other liquids or liquid metals are not different enough to those considered above to allow significantly smaller values of $\varepsilon$ ). This might explain why only the first two patterns in figure 14 were obtained by Mollot et al. (1993) for low-viscosity liquids. The situation is different if we excite high-order modes (Nicolás et al. 1996a,b), significantly lower the slenderness $A$ or increase the radius of the disks. In space platforms, $R$ may be as large as $5 \mathrm{~cm}$; if, for example, mercury is used and $|A|=17$, then $C=8.5 \times 10^{-5}, \varepsilon=0.024$ and $k$ may be as large as 2000 ; then the last two patterns in figure 14 can be also obtained.

\section{Concluding remarks}

We have considered the weakly nonlinear axisymmetric, nearly inviscid oscillations in a liquid bridge resulting from almost-resonant, axial vibrations of the disks. A cubic amplitude equation has been derived for the complex amplitude of the oscillating pressure and velocity fields and of the oscillating shape of the interface. That equation is a balance between inertia, damping, detuning from resonance, nonlinearity and forcing, and includes a second approximation in the damping term (in order to ensure a quantitatively good approximation for realistically large values of the capillary Reynolds number) and a first approximation of the remaining effects.

The coefficients of the amplitude equation have been calculated in terms of the slenderness and the inviscid mode being excited, and the large-time behaviour of the solutions have been discussed. In particular, we predict the liquid bridge to exhibit periodic oscillations when either only one disk is vibrated or both disks are vibrated with the same frequency. Typical response diagrams in terms of the forcing frequency exhibit hysteresis when the forcing amplitude is not too small; this hysteresis has not been detected experimentally (perhaps because most experiments in the literature seem to have been designed to confirm linear theories that do not predict that effect), but some recent experiments with pendant drops (De Paoli et al. 1995) showed the effect. If both disks are vibrated with different (but appropriately close) frequencies then we predict that generically the capillary bridge exhibits quasi-periodic oscillations with two frequencies, one of them almost equal to the forcing frequencies and the other one of the order of the difference between them. Typical response diagrams now exhibit multiplicity and period doublings that, again, have not been detected experimentally.

The Stokes boundary layers near the disks produce a steady (or slowly varying) forcing radial velocity that has been also calculated. The resulting slowly varying flow have been considered in $\S 5$, where some experimental observations by Mollot et al. (1993) have been explained and other non-observed flow patterns have been predicted. Also, the combined effect of the steady forcing terms due to vibration and thermocapillary stress have been analysed in $\$ 5.1$, to conclude that this streaming flow is not effective in counterbalancing thermocapillary convection. But our results suggested how to get the required effect (see Nicolás et al. 1996a,b).

A remarkable feature is that the amplitude equation giving the leading-order oscillating flow is decoupled from the streaming flow. This is an exclusive property of the axisymmetric case. The extension of the results in this paper to the nonaxisymmetric case leads to a more complex model that includes the coupled effect of two counter-rotating waves and a slowly varying flow (Higuera 1996). 
Finally, let us recall that our analysis requires several limitations that were discused in $\S 3.5$.

Numerical calculations in Sections 4.2 and 5 were performed by Mr. Marcos Vera and Dr. Damián Rivas respectively. We are also indebted to Dr. Rivas for helpful discussions, and to the three anonimous referees for several criticisms and suggestions on an earlier version of the paper that led to a significant improvement in the presentation. This research was supported by DGICYT and by the EEC Program on Human Capital and Mobility, under Grants PB-93-0046 and CHRX-CT-93-0413.

\section{Appendix.}

In this Appendix we give several groups of algebraic expressions that were omitted in $\S 3$ to facilitate the reading of this section. The first group deals with the integral expressions appearing in the solvability conditions $(3.31 b)$, which may be written as

$$
\begin{aligned}
& I_{2}=\delta \int_{-\Lambda}^{\Lambda} \int_{0}^{1}\left(U_{0} u_{\tau}+W_{0} w_{\tau}\right) r \mathrm{~d} r \mathrm{~d} z \\
& -\delta \int_{-\Lambda}^{\Lambda}\left[f_{\tau} f Q_{0}+(f-1) f_{\tau} Q_{0 r}-(f-1)\left(U_{0} u_{\tau}+W_{0} w_{\tau}\right)\right]_{r=1} \mathrm{~d} z \\
& +2 \varepsilon C\left\{H_{1} \mathrm{e}^{\mathrm{i} \Omega t} \int_{0}^{1} \int_{-\infty}^{0} U_{0}(r, \Lambda)\left[\widetilde{U}_{0}(r, \xi)-U_{0}(r, \Lambda)\right] r \mathrm{~d} r \mathrm{~d} \xi+\text { c.c. }\right\}+\text { HOT, } \\
& I_{3}=-C \varepsilon A \mathrm{e}^{\mathrm{i} \Omega t} \int_{-\Lambda}^{A} \int_{0}^{1}\left[\left(U_{0 r}^{2}+U_{0 z}^{2}+W_{0 r}^{2}+W_{0 z}^{2}\right) r+U_{0}^{2} / r\right] \mathrm{d} r \mathrm{~d} z+\mathrm{NRT}+\mathrm{HOT}, \\
& I_{4}=\int_{-A}^{A}\left\{\varepsilon\left(f W_{0}(1, z)+(f-1) W_{0 r}(1, z)\right)\left(A W_{0}^{*}(0, z) \mathrm{e}^{\mathrm{i} \Omega t}+\text { c.c. }\right)+\mathrm{i} \Omega(1-f / 2) F_{0} f_{t}\right. \\
& +(f-1)\left[f_{t}\left(U_{0 r}(1, z)+U_{0}(1, z)\right)-f Q_{0 r}(1, z)+F_{0}+F_{0}^{\prime \prime}\right] \\
& \left.-h(f-1)^{2}\left(F_{0}^{\prime \prime}+F_{0}^{\mathrm{iv}}\right)\right\} f_{t} \mathrm{~d} z-\mathrm{i} \Omega \int_{-A}^{A}\left\{(f-1)^{2}\left(1-\Omega^{2} f\right)+(f-1) f_{z z}+\frac{3}{2} f_{z}^{2}\left(1+f_{z z}\right)\right. \\
& \left.+\frac{1}{2} \varepsilon^{2} f\left(W_{0}^{*}(0, z) \mathrm{e}^{\mathrm{i} \Omega t}+\text { c.c. }\right)^{2}\right\} F_{0} \mathrm{~d} z+\int_{-A}^{A}\left[U_{0 r}(f-1)-f_{z} W_{0}\right]_{r=1}\left\{f-1+f f_{z z}+\frac{1}{2} f_{z}^{2}\right. \\
& \left.-\frac{1}{2}\left[f_{t}^{2}+\varepsilon^{2}\left(A W_{0}^{*}(0, z) \mathrm{e}^{\mathrm{i} \Omega}+\text { c.c. }\right)^{2}\right]\right\} \mathrm{d} z+\frac{\Omega^{2}}{2} \int_{-A}^{A}(f-1)^{3} U_{0 r}(1, z) \mathrm{d} z \\
& +\int_{-A}^{\Lambda}\left[U_{0 r r}(f-1)^{2} / 2-W_{0 r} f_{z}(f-1)\right]_{r=1}\left(f-1+f_{z z}\right) \mathrm{d} z-C \int_{-\Lambda}^{A} f_{t z} W_{0}(1, z) \mathrm{d} z \\
& +C \int_{-\Lambda}^{A}\left[f_{t}+\varepsilon\left(A W_{0 z}^{*}(0, z) \mathrm{e}^{\mathrm{i} \Omega t}+\text { c.c. }\right)\right] U_{0}(1, z) \mathrm{d} z+\text { HOT, } \\
& I_{5}^{ \pm}=\left(\mathrm{i} \Omega \mu \beta_{ \pm} \mathrm{e}^{\mathrm{i}\left(\Omega t+\omega_{ \pm} \tau\right)}+\text { c.c. }\right) \int_{0}^{1} r Q_{0}(r, \pm \Lambda) \mathrm{d} r \\
& \pm C^{1 / 2} \varepsilon \int_{0}^{1} U_{0}(r, \Lambda)\left[A \widetilde{U}_{0 \xi}(r, 0) \mathrm{e}^{\mathrm{i} \Omega t}+\text { c.c. }+C^{1 / 2} \tilde{u}_{1 \xi}(r, 0)+\varepsilon \tilde{u}_{2 \xi}(r, 0)\right] r \mathrm{~d} r+\text { HOT, }
\end{aligned}
$$

where $u$ and $w$ (the velocity components in the bulk) and $f$ are as given in (3.1), $\widetilde{U}_{0}, \widetilde{W}_{0}, \tilde{u}_{1}, \tilde{u}_{2}$ and $W_{0}^{*}$ are as given in (3.16)-(3.18) and (3.25), NRT stands for non-resonant terms (depending on the fast time variable as exp(imt), with $m \neq 1$ 
being an integer), and

$$
\mathrm{HOT}=o\left(\mu+\varepsilon C+\varepsilon^{2} C^{1 / 2}+\varepsilon^{3}\right) .
$$

Also, in order to obtain (A1)-(A3) we have used the solution in the Stokes boundary layer near $z=-\Lambda$ (not given in $\S 3.2$ ), the facts that $U_{0 z}(r, \Lambda) \equiv W_{0 r}(r, \Lambda) \equiv 0$, that the functions $U_{0}^{2}$ and $U_{0} W_{0 z}$ are even in the $z$-variable and that $W_{0}^{*}(0, z)=W_{0}(1, z)$ is purely imaginary and $F_{0}$ is real (see (3.5), (A9a) and (A9b); also, in order to obtain (A 3) we have used the equation

$$
\mathrm{i} \Omega \int_{-\Lambda+h_{-}}^{\Lambda+h_{+}}(f-1) f Q_{0}(1, z) \mathrm{d} z=-\mathrm{i} \Omega \int_{-\Lambda}^{\Lambda}\left[f(f-1)+2 f_{z}^{2}+(2 f-1) f_{z z}\right] F_{0} \mathrm{~d} z+o(\varepsilon \mu),
$$

which is obtained upon substitution of (2.9), (2.11), (3.7) and (3.8), and integration by parts.

The linear eigenvalue problem (3.5)-(3.8), may be solved in a semi-analytical form, as first shown by Sanz (1985). The eigenfrequencies are the real solutions of one of the following equations:

$$
\Lambda \tan \Lambda=\sum_{n \text { odd }} a_{n} r_{n} \quad \text { or } \quad \Lambda \cot \Lambda+\sum_{n \text { even }} a_{n} r_{n}=0,
$$

where

$$
\begin{aligned}
& a_{0}=1, \quad a_{n}=2 \Omega^{2} /\left(\Omega^{2} q_{n}-s_{n}\right) \quad \text { if } n \geqslant 1, \\
& q_{n}=I_{0}\left(l_{n}\right), \quad r_{n}=q_{n} /\left(l_{n}^{2}-1\right), \quad s_{n}=l_{n}\left(l_{n}^{2}-1\right) I_{1}\left(l_{n}\right), \quad l_{n}=n \pi / 2 \Lambda \quad \text { if } n \geqslant 0,
\end{aligned}
$$

and $I_{0}$ and $I_{1}$ are the first two modified Bessel functions. If the first equation of (A 6) holds (odd modes), $Q_{0}$ and $F_{0}$ are given (up to a common constant factor) by

$$
Q_{0}=\sum_{n \text { odd }} a_{n} I_{0}\left(l_{n} r\right) \cos \left[l_{n}(z+\Lambda)\right], \quad F_{0}=\Lambda \sin z / \cos \Lambda+\sum_{n \text { odd }} a_{n} r_{n} \cos \left[l_{n}(z+\Lambda)\right],
$$

while if the second equation of (A 6) holds (even modes), then

$$
Q_{0}=\sum_{n \text { even }} a_{n} I_{0}\left(l_{n} r\right) \cos \left[l_{n}(z+\Lambda)\right], \quad F_{0}=\Lambda \cos z / \sin A+\sum_{n \text { even }} a_{n} r_{n} \cos \left[l_{n}(z+\Lambda)\right]
$$

$U_{0}$ and $W_{0}$ are readily calculated by means of the second and third equations of (3.5).

The functions $Q_{1}$ and $F_{1}$ appearing in (3.37)-(3.39) are as given by

$$
\begin{aligned}
& Q_{\mathrm{I}}=-(1-\mathrm{i})\left[b Q_{0}+\partial Q_{0} / \partial \Lambda\right]+\sum_{n \text { odd }} b_{n} I_{0}\left(l_{n} r\right) \cos \left[l_{n}(z+\Lambda)\right] /(2 \Omega)^{1 / 2}, \\
& F_{1}=-(1-\mathrm{i})\left[b F_{0}+\partial F_{0} / \partial \Lambda+2 \Lambda\left(\partial \Omega / \partial \Lambda-\alpha_{1}(2 \Omega)^{1 / 2} \sin z /(\Omega \cos \Lambda)\right]\right. \\
& +\sum_{n \text { odd }} b_{n} r_{n} \cos \left[l_{n}(z+\Lambda)\right] /(2 \Omega)^{1 / 2}
\end{aligned}
$$

if $\Omega$ is a solution of the first equation of (A 6), or

$$
\begin{aligned}
& Q_{1}=-(1-\mathrm{i})\left[b Q_{0}+\partial Q_{0} / \partial \Lambda\right]+\sum_{n \text { even }} b_{n} I_{0}\left(l_{n} r\right) \cos \left[l_{n}(z+\Lambda)\right] /(2 \Omega)^{1 / 2} \\
& F_{1}=-(1-\mathrm{i})\left[b F_{0}+\partial F_{0} / \partial \Lambda+2 \Lambda\left(\partial \Omega / \partial \Lambda-\alpha_{1}(2 \Omega)^{1 / 2}\right) \cos z /(\Omega \sin \Lambda)\right] \\
& \quad+\sum_{n \text { even }} b_{n} r_{n} \cos \left[l_{n}(z+\Lambda)\right] /(2 \Omega)^{1 / 2}
\end{aligned}
$$


if $\Omega$ satisfies the second equation of (A 6); $U_{1}$ and $W_{1}$ are

$$
U_{1}=\left[i Q_{1 r}+(1-\mathrm{i}) \alpha_{1} U_{0}\right] / \Omega, \quad W_{1}=\left[\mathrm{i} Q_{1 z}+(1-\mathrm{i}) \alpha_{1} W_{0}\right] / \Omega .
$$

Here the constant $b_{n}$ is given by

$$
b_{n}=2 \Omega\left(\partial \Omega / \partial A-\alpha_{1}(2 \Omega)^{1 / 2}\right) q_{n} a_{n} /\left(\Omega^{2} q_{n}-s_{n}\right) \quad \text { for } n=0,1, \ldots,
$$

and the constants $a_{n}, l_{n}, q_{n}, r_{n}$ and $s_{n}$ are as given in (A 7)-(A 8). The results in the paper (i.e. the coefficients $H_{3}, H_{4}$ and $H_{5}$ of the amplitude equation $(3.3 b)$ ) do not depend on the constant $b$. The remaining functions appearing in (3.37)-(3.39) are given by

$$
\begin{aligned}
& Q_{22}=2 \Omega^{2} c_{0}+4 \Omega^{2} \sum_{k=1}^{\infty} c_{2 k} I_{0}\left(2 l_{k} r\right) \cos \left[2 l_{k}(z+\Lambda)\right] \\
& F_{22}=\mathrm{i}\left[F_{0}^{\prime}(z) W_{0}(1, z)-F_{0}(z) U_{0 r}(1, z)\right] / 2 \Omega+2 \sum_{k=1}^{\infty} c_{2 k} l_{k} I_{1}\left(2 l_{k}\right) \cos \left[2 l_{k}(z+\Lambda)\right],(A) \\
& U_{22}=\mathrm{i} Q_{22 r} / 2 \Omega, \quad W_{22}=\mathrm{i} Q_{22 z} / 2 \Omega \\
& Q_{20}=D_{3}-g_{0} / 2 \\
& F_{20}=D_{4} \cos z-D_{3}-\sum_{k=1}^{\infty} g_{2 k}\left(1-4 l_{k}^{2}\right)^{-1} \cos \left[2 l_{k}(z+\Lambda)\right]
\end{aligned}
$$

where

$$
\begin{gathered}
D_{1}=\int_{-A}^{\Lambda} F_{0}(z)^{2} \mathrm{~d} z / 4 \Lambda+\sum_{k=1}^{\infty}\left[s_{2 k} e_{2 k}+2\left(1-4 l_{k}^{2}\right) d_{2 k} \Omega^{2} q_{2 k}\right] /\left(4 \Omega^{2} q_{2 k}-s_{2 k}\right)\left(1-4 l_{k}^{2}\right) \\
D_{2}=\Lambda \cot \Lambda-1+8 \Omega^{2} \sum_{k=0}^{\infty} r_{2 k} /\left(4 \Omega^{2} q_{2 k}-s_{2 k}\right), \\
D_{3}=\left[\tan \Lambda \sum_{k=1}^{\infty}\left(1-4 l_{k}^{2}\right)^{-1} g_{2 k}+\int_{-\Lambda}^{A}\left|F_{0}(z)\right|^{2} \mathrm{~d} z / 2\right] /(\Lambda-\tan \Lambda), \\
D_{4}=\left[\Lambda \sum_{k=1}^{\infty}\left(1-4 l_{k}^{2}\right)^{-1} g_{2 k}+\int_{-\Lambda}^{\Lambda}\left|F_{0}(z)\right|^{2} \mathrm{~d} z / 2\right] /(\Lambda \cos \Lambda-\sin \Lambda),
\end{gathered}
$$

and for $n \geqslant 0$, the constants $l_{n}, r_{n}, q_{n}$ and $s_{n}$ are as given in (A 8), while $c_{n}, d_{n}, e_{n}$ and $g_{n}$ are as given by

$$
\begin{aligned}
& c_{n}=\left[2 D_{1} / D_{2}+\left(1-l_{n}^{2}\right) d_{n} / 2+e_{n}\right] /\left(4 \Omega^{2} q_{n}-s_{n}\right), \\
& d_{n}=-\mathrm{i} \int_{-\Lambda}^{A}\left[F_{0}^{\prime}(z) W_{0}(1, z)-F_{0}(z) U_{0 r}(1, z)\right] \cos \left[l_{n}(z+\Lambda)\right] \mathrm{d} z /(\Lambda \Omega), \\
& e_{n}=\int_{-\Lambda}^{\Lambda}\left[\left(2-3 \Omega^{2}\right) F_{0}(z)^{2}+W_{0}(1, z)^{2}-F_{0}^{\prime}(z)^{2}\right] \cos \left[l_{n}(z+\Lambda)\right] \mathrm{d} z / 2 \Lambda, \\
& g_{n}=\int_{-\Lambda}^{\Lambda}\left[\left(\Omega^{2}-2\right)\left|F_{0}(z)\right|^{2}-\left|W_{0}(1, z)\right|^{2}+\left|F_{0}^{\prime}(z)\right|^{2}\right] \cos \left[l_{n}(z+\Lambda)\right] \mathrm{d} z / \Lambda .
\end{aligned}
$$

Notice that as $2 \Omega$ approaches a solution of the second equation of (A 6), $D_{2} \rightarrow 0$ and $U_{22}, W_{22}, Q_{22}$ and $F_{22}$ diverge; also, as $A-\tan A \rightarrow 0$ (i.e. as a solution of the second 
equation of (A 6) approaches the solution $\Omega=0$ ) $D_{3} \rightarrow \infty, D_{4} \rightarrow \infty$ and $Q_{20}$ and $F_{20}$ diverge.

\section{REFERENCES}

Anilkumar, A. V., Grugel, R. N., Shen, X.P., Lee, C.P. \& Wang, T. G. 1993 Control of thermocapillary convection in a liquid bridge by vibration. J. Appl. Phys. 73, 4165-4170.

BATCheLOR, G. K. 1967 Introduction to Fluid Dynamics. Cambridge University Press.

Borkar, A. \& TSAmopoulos, J. 1991 Boundary layer analysis of the dynamics of axisymmetric capillary bridges. Phys. Fluids A 3, 2866-2874.

Brown, R. 1988 Theory of transport processes in single crystal growth from the melt. AIChE J. 34, $881-911$.

CHEN, T.Y. \& Tsamopoulos, J. A. 1993 Nonlinear dynamics of capillary bridges: theory, J. Fluid Mech. 255, 373-409.

Chen, T. Y., Tsamopoulos, J.A. \& Good, R. J. 1992 Capillary bridges between parallel and nonparallel surfaces and their stability. J. Colloid Interface Sci. 151, 49-69.

DePaOLi, D. W., Feng, J.Q., Basaran, O. A. \& ScOTt, T. C. 1995 Hysteresis in forced oscillations of pendant drops. Phys. Fluids 7, 1181-1183.

Fowle, A. A., WANG, C.A. \& Strong, P.F. 1979 Experiments on the stability of conical and cylindrical columns at low Bond numbers. Arthur D. Little Co.

Grasman, J. 1987 Asymptotic Methods for Relaxation Oscillations and Applications. Springer-Verlag.

HiguerA, M. 1996 Oscilaciones Débilmente no Lineales en Puentes Liquidos no Axilsimétricos. Doctoral Thesis, Universidad Politécnica de Madrid. In progress.

Higuera, M., Nicolás, J. A. \& VeGA, J. M. 1994 Linear oscillations of weakly dissipative axisymmetric liquid bridges. Phys. Fluids A 6, 438-450.

JURISH, M. \& LöSER, W. 1990 Analysis of periodic non-rotational $W$ striations in $M_{0}$ single crystals due to non-steady thermocapillary convection. J. Cryst. Growth 102, 214-222.

Kamotan, Y.S., OSTRACH, S. \& VARGAS, M. 1984 Oscillatory thermocapillary convection in a simulated floating-zone configuration. J. Cryst. Growth 66, 83-90.

Keller, H. B. 1987 Lectures on Numerical Methods in Bifurcation Problems. Tata Institute of Fundamental Research, Bombay.

LAPLACE, P.S. 1805 Theory of Capillary Attractions, supplement to the Tenth Book of Celestial Mechanics. Reprinted by Chelsea, 1966.

MANCEBO, F.J., Nicolás, J.A. \& VEGA, J.M. 1996 Chaotic mechanical oscillations in a nearlyinviscid liquid bridge at second-order 2:1 resonance, in preparation.

MEI, C. C. \& LIU, L.F. 1973 The damping of surface gravity waves in a bounded liquid. J. Fluid Mech. 59, 239-256.

Melrose, J.C. 1966 Model calculations for capillary condensation. AIChE J. 12, 986-994.

MESEGUER, J. 1983 The breaking of axisymmetric slender liquid bridges. J. Fluid Mech. 130, 123-151.

Meseguer, J. \& Perales, J. M. 1991 A linear analysis of g-jitter effects on viscous cylindrical liquid bridges. Phys. Fluids A 3, 2332-2336.

Mollot, D. J., Tsamopoulos, J., Chen, T. Y. \& Ashgriz, N. 1993 Nonlinear dynamics of capillary bridges: experiments. J. Fluid Mech. 255, 411-435.

NicoLÁs, J.A. 1992 Hydrodynamic stability of high-viscosity cylindrical liquid bridges. Phys. Fluids A $4,1-7$.

NiCOLÁs, J.A. \& VEGA, J.M. 1996 Linear viscous oscillations of axisymmetric liquid bridges, in preparation.

Nicolás, J.A., RIVAS, D. \& VEGA, J. M. $1996 a$ The interaction of thermocapillary convection and low-frequency vibration in nearly-inviscid liquid bridges. Z. Angew. Math. Phys. (submitted).

Nrcolás, J. A., Rivas, D. \& VeGA, J. M. $1996 b$ On the steady streaming flow due to high-frequency vibration in nearly-inviscid liquid bridges. J. Fluid Mech. (submitted).

PERALES, J. M. \& MESEGUER, J. 1992 Theoretical and experimental study of vibration of axisymmetric viscous liquid bridges. Phys. Fluids A 4, 1110-1130.

Perko, L. 1991 Differential Equations and Dynamical Systems. Springer-Verlag

Plateau, J. A. F. 1849 Sur les figures d'equilibre d'une masse liquide sans pesanteur. Mem. Acad. $R$. Belgique, Nonv. Ser. 23. 
PlateaU, J.A.F. 1863 Experimental and theoretical researches on the figures of equilibrium of a liquid mass withdrawn from the action of gravity. Transl. in Ann. Rep. Smithsonian Inst. (1863-1866) 207-285.

Preiser, F., SchwaBe, D. \& Sharman, A. 1983 Steady and oscillatory thermocapillary convection in liquid columns with free cylindrical surface. $J$. Fluid Mech. 126, 545-567.

RAYLBIGH, LoRD 1879 On the instability of jets. Proc. London Math. Soc. 10, 4-13.

RAYLEIGH, LORD 1892 On the stability of cylindrical fluid surfaces. Phil. Mag. 34, 177-180.

RIVAS, D. \& MESEGUER, J. 1984 One-dimensional self-similar solution of the dynamics of axisymmetric slender liquid bridges $J$. Fluid Mech. 138, 417-429.

SANZ, A. 1985 The influence of the outer bath in the dynamics of axisymmetric liquid bridges. $J$. Fluid Mech. 156, 101-140.

SANZ, A. \& DíEZ, J. L. 1989 Non-axisymmetric oscillation of liquid bridges. J. Fluid Mech. 205, 503-521.

SchULKES, R. M. S. M. 1993 a Nonlinear dynamics of liquid columns: A comparative study. Phys. Fluids A 5, 2121-2130.

SCHULKes, R. M.S. M. $1993 b$ Dynamics of liquid jets revisited. J. Fluid Mech. 250, 635-651.

Tsamopoulos, J., Chen, T. \& BoRKar, A. 1992 Viscous oscillations of capillary bridges. J. Fluid Mech. 235, 579-609.

UrSell, F. 1952 Edge waves on a sloping beach. Proc. R. Soc. Lond. A 214, 79-97.

Young, X. 1805 Essay on the cohesion of fluids. Phil. Trans. R. Soc. Lond. A 306, 347-370.

ZHANG, Y. \& AlEXANDER, J.I.D. 1990 Sensitivity of liquid bridges subjected to axial residual acceleration. Phys. Fluids A 2, 1966-1974.

Zasadzinski, J.N., Sweeney, J. B., Davis, H. T. \& SCRIVEn, L. E. 1987 Finite element calculation of fluid menisci and thin-films in model porous media. J. Colloid Interface Sci. 119, 108-116. 